\title{
Emerging ExG-based NUI Inputs in Extended Realities: A Bottom-up Survey
}

\author{
KIRILL A. SHATILOV and DIMITRIS CHATZOPOULOS, Hong Kong University \\ of Science and Technology, Hong Kong \\ LIK-HANG LEE, KAIST, Republic of Korea and University of Oulu, Finland \\ PAN HUI, Hong Kong University of Science and Technology, Hong Kong and University of Helsinki, Finland
}

Incremental and quantitative improvements of two-way interactions with extended realities (XR) are contributing toward a qualitative leap into a state of XR ecosystems being efficient, user-friendly, and widely adopted. However, there are multiple barriers on the way toward the omnipresence of XR; among them are the following: computational and power limitations of portable hardware, social acceptance of novel interaction protocols, and usability and efficiency of interfaces. In this article, we overview and analyse novel natural user interfaces based on sensing electrical bio-signals that can be leveraged to tackle the challenges of XR input interactions. Electroencephalography-based brain-machine interfaces that enable thought-only hands-free interaction, myoelectric input methods that track body gestures employing electromyography, and gaze-tracking electrooculography input interfaces are the examples of electrical bio-signal sensing technologies united under a collective concept of ExG. ExG signal acquisition modalities provide a way to interact with computing systems using natural intuitive actions enriching interactions with XR. This survey will provide a bottom-up overview starting from (i) underlying biological aspects and signal acquisition techniques, (ii) ExG hardware solutions, (iii) ExG-enabled applications, (iv) discussion on social acceptance of such applications and technologies, as well as (v) research challenges, application directions, and open problems; evidencing the benefits that ExG-based Natural User Interfaces inputs can introduceto the areaof XR.

CCS Concepts: • Human-centered computing $\rightarrow$ Mixed/augmented reality; Graphical user interfaces; - Software and its engineering $\rightarrow$ Development frameworks and environments; • Hardware $\rightarrow$ Biologyrelated information processing

Additional Key Words and Phrases: Mobile augmented reality, user interactions, Electromyography, Electroencephalography, Silent Speech Interfaces, Extended Reality

ACM Reference format:

Kirill A. Shatilov, Dimitris Chatzopoulos, Lik-Hang Lee, and Pan Hui. 2021. Emerging ExG-based NUI Inputs in Extended Realities: A Bottom-up Survey. ACM Trans. Interact. Intell. Syst. 11, 2, Article 10 (July 2021), 48 pages.

https://doi.org/10.1145/3457950

The reviewing of this article was managed by associate editor Tian, Feng.

This research has been supported in part by 5G-VIIMA and REBOOT Finland IoT Factory projects funded by Business Finland, the 6G Flagship project funded by the Academy of Finland (Decision No. 318927), the 5GEAR project and FIT project from the Academy of Finland, project 16214817 from the Research Grants Council of Hong Kong, as well as Hong Kong Ph.D. Fellowship Scheme.

Authors' addresses: K. A. Shatilov, Hong Kong University of Science and Technology, Hong Kong; email: kshatilov@connect.ust.hk; D. Chatzopoulos, Hong Kong University of Science and Technology, Hong Kong; email: dcab@ cse.ust.hk; L.-H. Lee, KAIST, Republic of Korea and University of Oulu, Finland; email: likhang.lee@kaist.ac.kr; P. Hui, Hong Kong University of Science and Technology, Hong Kong and University of Helsinki, Finland; email: panhui@ust.hk.

This work is licensed under a Creative Commons Attribution International 4.0 License.

(C) 2021 Copyright held by the owner/author(s).

2160-6455/2021/07-ART10 \$15.00

https://doi.org/10.1145/3457950 

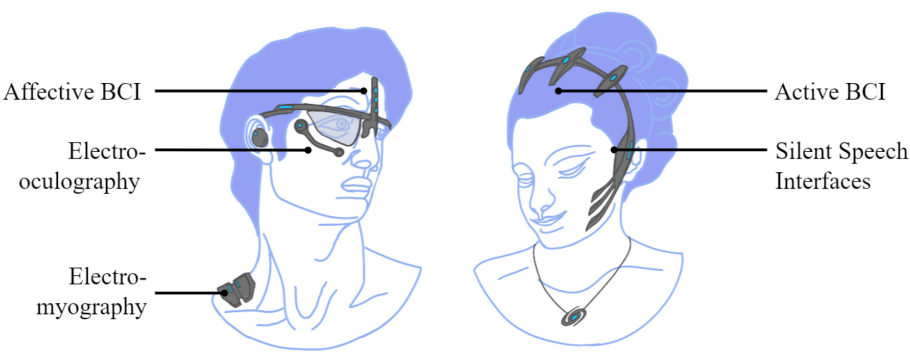

Conceptual illustrations of ExG-based natural user interfaces that transform collected signals from brain $(\mathrm{BCl})$, muscles (electromyography and Silent Speech), and eye movements (electrooculography) to inputs on connected interactive systems.

\section{INTRODUCTION}

The interaction experience with real, semi-virtual, and virtual worlds in stationary and mobile augmented reality (AR), mixed reality (MR), and virtual reality (VR) can be consolidated under the term of extended realities (XR) [147] (see Figure 1). Driven by the industry leaders [49, 90], mass consumer adoption [95, 141] and academic researchers [109, 198], XR fulfills demand arising in multitudinous areas of entertainment [98], education and training [81], medicine [87], office [97], and everyday life [71]. However, wide adoption of XR is held back by many factors: usability and efficiency of interfaces [131], computational and power limitations of hardware [71], social acceptance of novel interaction protocols [111,193].

The two-way interaction between users and XR devices is not thoroughly designed. On the one hand, many micro-interaction approaches have been designed for displaying information to the users. Apart from evolving display technologies, wearable devices such as smartwatches can vibrate when the user is receiving a phone call or display the beginning of a message on their screen. However, the input approaches enabling users to interact with the devices are limited to touchscreens and physical controllers. In the case of mobile interactions, reacting to notifications and dismissing them or scrolling application feed relies on touchscreen inputs. In the case of stationary VR, the interaction with virtual worlds is constrained by obtrusive physical controllers. The increasing adaptation of wearable computers with limited screen real estate pose constraints to the users' inputs, where the users cannot manipulate digital objects directly [133].

When using desktops and laptops, users rely on bulky and tangible interfaces such as mice, touchpads, and keyboards. As commented by Klemmer et al., such computers see humans as one-eyed, two-ear, and one-handed creatures [115], implying that two-way interactions are narrowed down to certain body parts and their capabilities. Information output primarily relies on the visual and auditory mediums, while the input on the graphical user interfaces relies exclusively on the users' hands. Therefore, it is necessary to explore alternative input approaches for XR.

In this article, we analyze novel input techniques based on sensing electrical bio-signals, such as electromyography (EMG), electrooculography (EOG), and electroencephalography (EEG), while discussing their applicability to XR and social acceptance aspects. All these techniques form the collective concept of ExG (EEG, EMG, EOG), and face similar challenges, such as the interference from external and internal noise, the challenge of energy and time-efficient analysis of the recorded signals, and many others [44]. We outline how these technologies can contribute toward more comprehensive input paradigms in XR and limitations that have to be overcome along the way. 


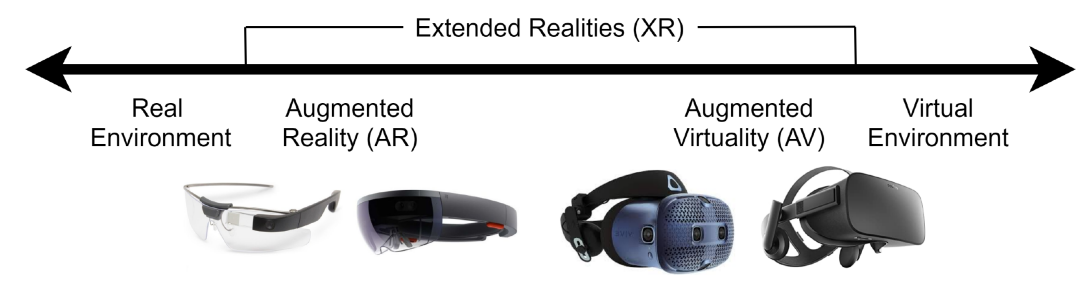

Fig. 1. Reality-virtuality continuum augmented by XR hardware examples (fLTR): Google glass, Microsoft Hololens, HTC Vive Cosmos, and Oculus Rift.

\subsection{Natural User Interfaces}

The book Brave NUI World gives a definition of Natural User Interfaces (NUIs) as truly natural by taking full advantage of the user's bandwidth, and the grand challenges are to design, research, and engineer such user interfaces with modern input technology (e.g., sensors) [213]. In that context, "natural" relates to actions performed by users, rather than any properties of the interfaces themselves; naturality ideally implies an immediate capability to interact with a system. NUIs function as natural extensions of a person's cognitive and/or physical abilities [107]. NUIs circumvent the flaws of traditional input/output $(\mathbf{I} / \mathbf{O})$ interfaces, not only by helping disabled people to interact with computing devices but also by augmenting individuals by new input methods and modalities to interact with mobile user interfaces, virtual and real worlds in scenarios where traditional controllers fail or are highly constrained. We introduce the following emerging ExG NUIs below.

Speech Interfaces. Speech-based input is a common example of natural user interfaces in the domain of human-computer interaction (HCI). Speech as an input modality is widely used in mobile devices with the most popular application to be voice assistants. However, spoken speech has several downsides as an input method with the most popular the downside of disclosing sensitive information to both the surrounding listeners and remote speech processing servers. The wide adoption of speech-based interfaces is also inhibited by the fact that devices can overhear any conversation in the vicinity (eavesdropping issue) and that speech-based input systems listen for everyone in the reachable area (impersonal devices issue) [111]. Silent speech interfaces (SSIs), however, can provide silent, concealed, and seamless ways of interacting with wearable devices and computer peripherals. SSI technology can be based on several modalities [88]: (i) video-based lip movement recognition, which has limited applicability in mobile computing; (ii) ultrasonic Doppler sensing; or (iii) decoding of facial muscles movements. SSIs can be used for high-speed input of words and phrases within limited vocabulary using lightweight signal acquisition hardware [111].

Gesture Inputs. Gesture-based inputs are the second example of NUI deployed in contemporary computing systems. It varies from the visual-based whole-body posture sensing, e.g., Microsoft Kinect [155], to the detection of precise finger configuration [129], which can be achieved by using ultrasound produced and detected by off-the-shelf devices' speakers and microphones [139] or WiFi signals [221]. The abundance of various sensors in wearable devices brought other ways of gesture inputs. Zhang et al. [230], for example, utilized a generic photoplethysmography (PPG) sensor, which is used in most of the smartwatches and bands to measure heart rate to identify gestures. However, most of the gesture-based input systems are sensitive to interference and are hard to deploy in mobile scenarios. Surface Electromyography (sEMG), a non-invasive method to quantitatively measure electrical activity in human muscles by estimating the electric potential differences between muscle and ground electrodes, offers a robust and convenient method to recognize gestures as well. EMG measurements and analysis is used for 
medical, rehabilitation, and sports purposes, alongside HCI and prosthesis control by utilizing non-obstructive, lightweight sensors with the capability of wireless signal transmission.

Gaze Interaction. Gaze tracking human-machine interfaces (HMIs) are a part of the NUI paradigm as well. Visual-based eye-tracking systems [42] can precisely estimate where a user looks on the screen of a personal computer or head-mounted display (HMD). Several solutions deploy small cameras on a frame of eyeglasses to track the eyesight, providing a tool suitable for mobile usage $[39,112]$. Alternatively, EOG, which measures corneo-retinal standing potential between the front and back parts of an eye, can be used to track the eye position.

Brain-Computer Interfaces. Another modality is the Brain-Computer Interfaces (BCIs) or Brain-Machine Interfaces (BMIs). They allow sending a command to a computer using only the power of thought. Historically developed to provide a communication tool for locked-in or physically challenged patients, BCIs are evolving to a technology for a general consumer market $[25,30,31,37]$. Modern non-invasive BCIs can provide a covert way of information input without any mechanical actions from a user [180].

Furthermore, different NUI modalities can be combined to build hybrid or multi-modal HMIs. Aiming to reduce the limitations of combined modalities, hybrid interfaces can achieve higher throughput rates, better usability, and universality [85, 225].

NUI modalities can be divided into two groups: affective and direct control. When an affective modality is employed, the user's mental state or physical condition is monitored. This information can be used to adopt a user interface [55], track the involvement into an activity [79], estimate emotional feedback $[178,200]$, and others. The second group, modalities of direct control are used to explicitly generate input signals, e.g., in the case of motor imagery control paradigm, a user imagines the execution of certain motor activities. The applications of direct control modalities spans from spellers [186], to control of drones [211] and Internet of Things (IoT) devices [229]. Direct control modalities can be further subdivided into exogenous and endogenous depending on the source of stimuli. Exogenous HMIs use external stimuli to induce a certain activity. SteadyState Evoked Visual Potentials (SSVEPs), a BCI paradigm when a set of flickers is presented to a user, eliciting a response in the Visual cortex, is an example of exogenous HMI. Endogenous interfaces rely on an internal source of a certain activity. Self-motivated gestures, speech, or motor imagination can serve as examples of endogenous direct control NUIs.

ExG-based natural user interfaces face multiple problems: (i) the nature of biological signal limits effective bandwidth; (ii) signals are extremely susceptible to interference; (iii) acquisition hardware can be bulky and inaccurate; and (iv) applications are limited. Moreover, the union of NUIs and mobile computing brings its own challenges: selection and combination of hardware, software development and interoperability, and competition for body space and energy needs.

\subsection{The Frontier of Interaction Design with Extended Realities (AR/MR/VR)}

Apart from the motivation of two-way interaction between users and devices in $\mathrm{XR}$, the research community has reflected that novel interaction paradigms are not widespread outside the laboratory settings because of the limited mobility [116]. The mobile sensors offer a foundation of including the crucial factor of mobility in the design space of input interaction for the frontier of immersive reality. Since the early 2010 s, three apparent trends have been observed in the context of HCI for the mobile inputs, which are presented in detail below.

First, the design of input approaches shifts from noticeable body movements toward subtle and concealed operations. As advocated by Wigdor and Wixon [213], touch-based and gestural interactions are proliferating, and eventually serve as the primary interaction approaches between humans and computers more than the desktop metaphor, namely the post-Windows, Icons, Menus, Pointers (WIMP) paradigm [106]. We observe a smaller and smaller degree of user 
movements for gestural inputs [52], in descending order, from the whole body [188], upper body gestures [216], arm gestures [74, 133], palm gestures [51], finger gestures [84], to a minimal of fingertip movements [129]. For example, the daily text entry scenarios for mobile augmented reality (MAR) utilize a smaller interaction space in mid-air, reducing from a square of standard QWERTY keyboard to a horizontal space of 1-line non-standard keyboard [133]. Also, the fingertip movements in mid-air can accomplish the pointing and selection of digital contents in MAR. Similarly, the location of touch-based inputs can proceed with some noticeable approaches, such as the spectacle frame of immersive reality headsets [220], handheld controller [223], as well as the surroundings of the headsets $[129,133]$. Recent works demonstrate more concealed operations for immersive reality in mobile environments, with on-body interaction [131], including but not limited to finger-to-forearm interaction [60], finger space interaction [132], and thumb-to-index finger interaction [218].

Second, interaction design input approaches leverage the innate ability of embodied interaction [203], to facilitate the user's interaction with the mobile devices. Examples include the resource inherited in the users in terms of humans' physical body, experiences, and skills [128], especially designated for the user interaction with immersive reality driven by mobile sensors. TiPoint [129], for example, leverages the dexterous fingertips as a pointing device for the direct acquisition of digital overlays in the spacious mid-air. Similarly, HIBEY [133] considers the advantages of ingrained knowledge of alphabetic order to enable users to achieve text entry in invisible layouts.

Third, the fact that human thumbs can reach other fingers is employed to formulate a keyboard within a palm area [132]. An additional example is that the input of muscle tension drives the manipulation of three-dimesional (3D) objects and the corresponding pseudo-haptic (visual) feedback [187].

As discussed above, user interactions with the MAR hardware have to consider the subtle and concealed operations [134]. Considering the four introduced NUIs, speech interfaces are not considered as a concealed and subtle channel. Among the remaining candidates, BCIs achieve more subtle and concealed operations than the gesture inputs and gaze interaction, as they rely on the electric currents detected in the inner body of the user. Also, the ExG-based interfaces are highly characterized by our innate ability and habits as reflected by our minds and muscle control. The exploration of ExG for a broad range of neurally based HCI applications is still at a nascent stage [150]. While some may argue that interaction with immersive realities is regarded as the "old wine in a new bottle" [58] the motivation of two-way interactions and the key trends of designing XR input solutions definitively demonstrate the novel challenges to be solved by ExG-based interfaces.

\subsection{Contributions and Methodology}

This survey reviews emerging ExG modalities: electroencephalography-based BCIs, EOG-based HCIs, and myoelectric (including Silent Speech) HCIs, which are all non-invasive signal acquisition techniques. Representative examples are listed in Figure 2. We follow a bottom-up approach to discuss the mentioned NUIs and their existing and potential applications. This survey aims to bring together XR and novel ExG-based NUIs, such as BCI, myoelectric, and gaze interfaces, and serve as a bridge between areas. In general, biological foundations of BCIs are discussed, for example, in Reference [182], paradigms design in Reference [186]; myoelectric control interfaces in Reference [171]; aspects of silent speech and its applications in References [110, 156, 192]; and hybrid interfaces in Reference [96]. Prior surveys attempt to illustrate the potential applications of $\mathrm{BCI}$ in immersive technologies (AR/VR) and robotics. They focus on the SSVEP in Virtual Reality [180], the combination of AR and robotics supported by several BCI research prototypes [198], as well as BCI-supported drone controls [168]. Other mobile computing surveys have reviewed 


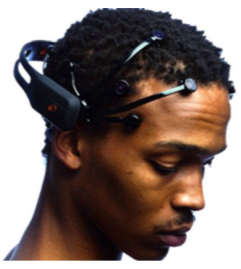

(a) Emotiv $\mathrm{BCl}[16]$

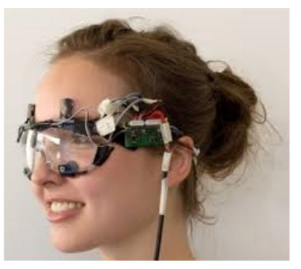

(b) EOG glasses from [68]

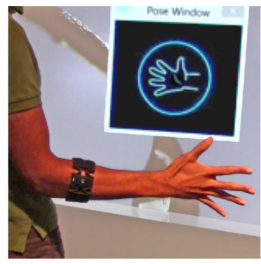

(c) Myopoint [92]

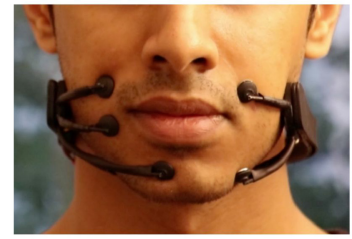

(d) SSI from [111]

Fig. 2. Examples of existing NUIs.

interaction techniques and their limitations for mobile systems: input methods in MAR [71]; limitations of user interactions with smartglasses [131]; haptic modality for mobile devices [62]; and hardware specifications and available sensors on smart wearables [173].

The primary contributions of this survey are summarized as follows. First, we outline the potential and existing NUI paradigms from the literature that can be effectively introduced to the challenges and future directions for designing ExG-based input methods for mobile devices. Second, the survey connects the biological mechanisms of ExG to the latest consumer-grade ExG wearable and corresponding research-prototype hardware. Third, the survey gives a comprehensive overview of NUI-enabled applications driven by ExG wearables for the user interaction in immersive realities, outlining potential scenarios, where XR can greatly benefit from the introduction of ExG as an input modality. Finally, this article presents an interview questionnaire with 93 participants to pinpoint the potentials of deploying such ExG-supported NUIs to immersive environments, resulting in positive feedback.

We design and follow the framework to establish the applicability of ExG-based interfaces in the area of mobile XR technology based on three main criteria: (1) information transfer rate (ITR), (2) Mobility, and (3) Social acceptance. The aim of the ExG-based interfaces is to map oscillating, endogenous or exogenous bio-electrical activity to commands for a computing system. The speed of this conversion, ITR, is defined as [194] ITR $=B / T$, where $T$ is an average time required to input a command, and $B$ is an average amount of bits, which in turn can be defined as follows:

$$
B=\log _{2} N+P \log _{2} P+(1-P) \log _{2} \frac{1-P}{N-1},
$$

where $N$ is a number of possible commands and $P$ is a selection accuracy $(1-P$ represents the error frequency). Information transfer rates define how effectively users can utilize certain interfaces and communicate with each other or with computing systems. The estimated ITR of human speech, for example, is roughly 40 to $60 \mathrm{bits} / \mathrm{s}$, or [2400;3600] bits/min (bpm) [185], while state-of-the-art BCI reaches $300 \mathrm{bpm}$ [161]. We use ITR numbers from the literature or calculate it ourselves based on the reported results (number of recognized labels, accuracy, time window) for the analysis. As for mobility, we access the signal acquisition hardware and its compatibility with mobile/stationary HMDs. Social acceptance is established by a questionnaire that is presented in Section 6. Additionally, we consider Non-intrusiveness constraint (a modality should not require the user's intent concentration on any input-related task), Non-invasiveness constraint (a modality should not rely on any invasive technique, e.g., brain implants that require surgery), and Non-obstructiveness constraint (a modality should not introduce additional physical hardware that might constrain the user's physical mobility).

The rest of this manuscript is organized as follows: Section 2 presents the biological and physical background of the discussed modalities. Section 3 discusses mobile hardware for signal acquisition, providing examples of portable consumer and research-grade devices. Section 4 provides an 


\begin{tabular}{|c|c|c|c|c|c|}
\hline \multicolumn{2}{|c|}{ Section 2} & Section 3 & Section 4 \& 5 & Section 6 & Section 7 \\
\hline $\begin{array}{l}\text { Biological } \\
\text { Foundations }\end{array}$ & $\begin{array}{c}\text { Signal } \\
\text { Acqusition }\end{array}$ & $\begin{array}{c}\text { NUI } \\
\text { Hardware }\end{array}$ & $\begin{array}{l}\text { Two-way } \\
\text { Interaction }\end{array}$ & $\begin{array}{c}\text { Social } \\
\text { Acceptance }\end{array}$ & $\begin{array}{c}\text { Future } \\
\text { directions }\end{array}$ \\
\hline $\begin{array}{l}\text { - } \mathrm{BCl} \\
-\mathrm{RCP} \\
\text { - Myoelectricity } \\
\text { - Speech }\end{array}$ & $\begin{array}{l}-E E G \\
-E O G \\
-E M G\end{array}$ & $\begin{array}{l}-E E G \\
-E O G \\
-E M G \\
-S S I\end{array}$ & $\begin{array}{l}\text { - EXG applications } \\
\text { in XR } \\
\text { - Reinforcement } \\
\text { of the interaction }\end{array}$ & $\begin{array}{l}\text { - Personal use } \\
\text { - Observer } \\
\text { acceptance }\end{array}$ & $\begin{array}{l}\text { - Challenges } \\
\text { - Redefining UX } \\
\text { - ExG adoption }\end{array}$ \\
\hline
\end{tabular}

Fig. 3. Structure of the survey.

overview of existing ExG-enabled applications followed up by a discussion on social acceptance of ExG-enabled NUIs 6. Section 8 concludes the survey outlining challenges, potential applications, and emerging usage scenarios. Additionally, we provide three appendices with detailed information regarding portable EEG headsets (Appendix A), wireless EMG sensors (Appendix B), and the questions and answers of the conducted questionnaire (Appendix $\mathrm{C}$ ). The structure of the survey is presented in Figure 3.

\section{BIOLOGICAL FOUNDATIONS}

In this section, we briefly introduce biological phenomena and mechanisms upon which various NUI modalities are based: from the human brain and its properties that can be used in affective computing and to input multiple control commands; cornea-retinal potential that is measured by EOG; to the nature of myoelectric currents in muscles and speech production. Additionally, we introduce concepts of signal acquisition techniques and metrics. We aim to outline NUI paradigms suitable for potential usage with mobile systems and unconstrained scenarios that can deliver acceptable signal quality according to the introduced metrics.

\subsection{BCI: Neurological Phenomena}

The human brain contains 100 billion neurons; each neuron is an electrically excitable cell that communicates with others through synapses. Interconnected neurons form synaptic, or neural networks [94]. Once the incoming charges from synaptic connections reaches a certain threshold, the neuron passes the signal forward; this process of in-cell electrical potential rising and falling is called action potential. Rhythmic patterns of action potentials of neurons in the brain are referred to as neural oscillations or brainwaves. Patterns of different frequencies are related to various types of activities. For example, oscillations at frequencies of $8-12 \mathrm{~Hz}$ are called alpha waves and are usually associated with the relaxed state of mind, meditation, or daydreaming; consciousness, alertness, and decision making are characterized by the frequency range of $12-25 \mathrm{~Hz}$, so-called beta waves [182]. Affective state BCIs are based on analyzing oscillation patterns by extracting dominant frequencies from the recorded brain activity.

The brain can be subdivided into the following regions known as the brain lobes: frontal, parietal, temporal, occipital. The outer layer of the brain, which is called cerebral cortex, can be divided into smaller areas, each of them is associated with a certain activity or function [182]. Lobes and several BCI-related areas are depicted in Figure 4: Motor Imagery- (MI) related potentials are observed in the motor cortex; visually evoked-in the visual cortex; attention-related potentials such as P$300-$ in the frontal lobe. 


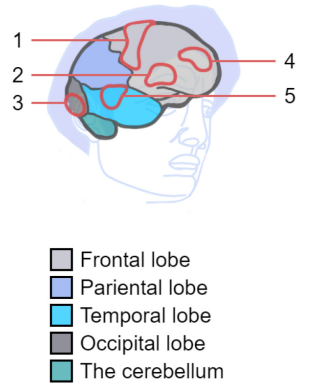

Fig. 4. Brain lobes and areas: 1, Motor cortex; 2, Speech area; 3, Visual cortex; 4, Concentration and planning; and 5, Language.

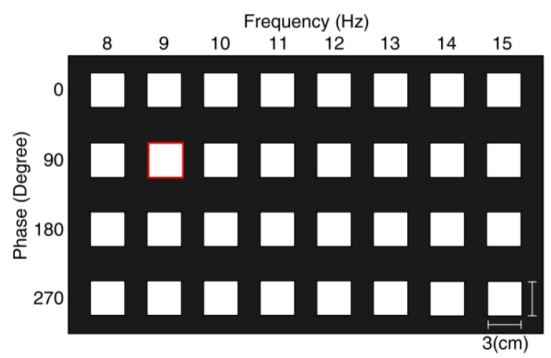

Fig. 5. SSVEPs input UI. A matrix of flickering markers from Reference [162].

Visually Evoked Potentials. Visually Evoked Potentials (VEP) provide the highest ITRs among contemporary BCIs [186]. BCIs based on VEPs belong to the group of attention-based BCIswhere the user has to focus on or pay advert attention to one of the presented stimuli. Such stimuli can be a set of flickering markers or multiple haptic devices vibrating on different frequencies. Attention-based BCIs are sometimes also referenced as Selective Attention BCIs [127]. SSVEPs are the potentials evoked in the human brain by attending visual stimuli flickering at some rate. If multiple stimuli flashing at different rates are presented to the patient, then the stimulus, that the patient attends to, will elicit a larger response amplitude than the stimuli that the user ignores. A typical SSVEPs interface with multiple stimuli is presented in Figure 5. Every flashing marker can represent buttons or other UI elements. SSVEPs are distinguishable when analyzing the frequency spectrum of the brain of both adults and children [167]. Usually, frequencies within the range [5; 50] $\mathrm{Hz}$ are used for controlling the flashing of markers. In Reference [167] 6.2, 7.7, and $10 \mathrm{~Hz}$ are used; another study [138] used 9 flickers of frequencies from 27 to $43 \mathrm{~Hz}$ with an incremental step of $2 \mathrm{~Hz}$. The precise values of frequencies are chosen to elicit higher and more distinguishable from each other responses; additionally, the chosen frequencies are less likely to elicit photo-induced seizures [167]. A bigger number of presented markers, and thus higher ITR, can be achieved using various frequencies, and different phases [162] at the same frequency. The time interval of a patient paying attention to a visual flicker required for accurate frequency detection is below $5 \mathrm{~s}[138,162$, 167].

SSVEP-based BCIs are widely adopted mostly due to the fact that they provide a high number of available input commands (up to 40 in Reference [161]) and require less concentration and mental efforts from users. However, constantly flashing, large, and spacious visual stimuli might be considered tiring and irrelevant to the spelling task itself making some users feel fatigued and annoyed [144]. To address this issue other types of visually evoked potentials are considered for usage 

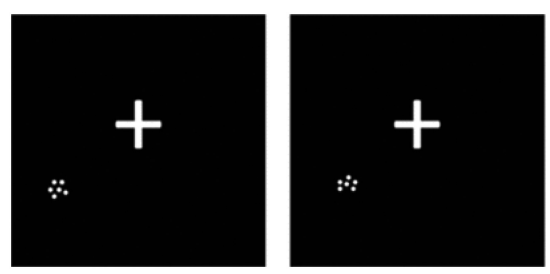

Fig. 6. aVEPs: The cross represents the gaze direction and points the stimulus itself (from Reference [217]).

\begin{tabular}{|c|c|c|c|c|c|c|c|}
\hline \multicolumn{2}{|c}{ N/A } & \multicolumn{2}{c}{ N/A } & \multicolumn{2}{c}{ N/A } & \multicolumn{2}{l}{$100 \mathrm{~cm}$} \\
A & B & C & D & E & F & G & H \\
\hline I & J & K & L & M & N & 0 & P \\
\hline Q & R & S & T & U & V & W & X \\
\hline Y & Z & Sp & 1 & 2 & 3 & 4 & 5 \\
\hline 6 & 7 & 8 & 9 & 0 &. & Ret & Bs \\
\hline$?$ & , & ; & I & I & + & - & Alt \\
\hline Ctrl & $=$ & Del & Home & UpAw & End & PgUp & Shift \\
\hline Save & - & F2 & LfAw & DnAw & RtAw & PgDn & Pause \\
\hline Caps & F5 & Tab & EC & Esc & email & ! & Sleep
\end{tabular}

Fig. 7. P-300 input UI [186].

in non-invasive BMIs, such as Miniature Asymmetric Visual Evoked Potentials (aVEPs) [217]. Miniature aVEPs arise when very small and inconspicuous asymmetric lateral visual stimuli are presented in the peripheral vision, which is an area outside of $2^{\circ}$ of the eccentricity of the human visual field. Presented peripheral stimulus subtends only $0.5^{\circ}$ of visual angle at an eccentricity of $2.1^{\circ}$ as depicted in Figure 6. Such a stimulus induces a different response in two hemispheres of the visual cortex, activating an area as small as $1.6 \mathrm{~mm}^{2}$ [217]. It is also claimed that such a stimulus will be more compatible with XR scenarios as it allows users to explore surroundings, observe the environment, and use interface components without a need to focus on task-irrelevant markers. However, it might be challenging to detect induced miniature potentials $(0.5 \mu \mathrm{V})$ using mobile EEG hardware.

Event-related Potentials. Another example of attention-based BCIs is the $P-300$ paradigm where a matrix of icons, which can be alphabetic symbols or any control UI elements, are presented to a user that focuses on one desired input. Each element of the matrix is intensified (or highlighted) in a random sequence. The elicited potential happens after $300 \mathrm{~ms}$ (hence the name) after the element is intensified. P-300 potential is most clearly observed in the Frontal lobe.

Motor Imagery. As an example of endogenous BCI, the MI paradigm relies on detecting asynchronous irregular patterns that emerge when humans willfully execute or imagine motor activity. Volitional imagination of moving a certain part of the body (tongue, left or right arm, or feet) can be tracked by the increased cortical activity in the area of Motor Cortex designated to control the involved muscles. MI-based BCIs can be used to input one of several commands (usually binary left/right) at a time, thus they have limited ITRs [127]. Usage of MI BCI is an intuitive approach to control drones or robotic manipulators: the desired direction of the movement can be perfectly harmonized with the direction of the imagined motor activity [168].

Electroencephalography. EEG measures the electrical charges of the synchronized synaptic activity through electrodes placed on the scalp; a typical electrode consists of a metal plate and 


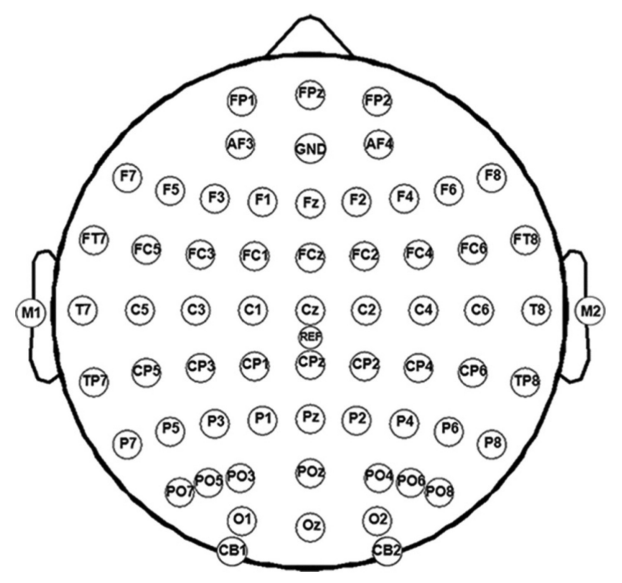

Fig. 8. Electrode locations according to the international 10-20 system (from Reference [217]).

an amplifier. To describe the electrode placement over the head, the 10-20 system is used, where electrodes are placed at distances of 10 or $20 \%$ of length between fixed reference points on the scalp (see Figure 8). The choice of electrode's location affects the BCI paradigms that can be used, for example, MI-related potentials are best detected on top of the scalp in the parietal area.

EEG has high temporal resolution and can detect changes in milliseconds, providing sampling frequencies up to $20 \mathrm{kHz}$ (typical sampling rate spans from 250 to $2 \mathrm{kHz}$ ). The fact, that hardware required for EEG measurements is affordable, has lead to its wide adoption and development. The potential of a single neuron is too small to measure using non-invasive techniques, thus a single electrode measures the sum of synaptic charges large enough to be propagated through different layers: brain itself, dura mater (three layers of the protective tissue around the brain), skull, skin, electrode gel (if applicable). The larger the pool of neurons involved in synchronous activity, the stronger the electric field they produce the clearer signal is acquired [105]. EEG is not devoid of limitations: Charges quantified on the scalp are smeared by the volume conduction effect; charge propagation is affected by the skin conductivity that depends on the number of dead cells, oil and water contents, sweat, and so on. A very important thing to consider in EEG systems is a noise factor: external coming from electrical devices around the recording hardware and internal noiserib cage expansion, breathing, blinking, EOG and EMG artifacts, any other kinds of movements. Passive shielding of room and cables alongside active electrodes (when the amplifier is placed as close as possible to the recording surface) are used to minimize external noise. For reducing internal noise experiments are run in a controlled environment and patient's behavior is strictly limited by experiment procedure. Additionally electrodes should settle first, allowing the potential of each electrode to reach a steady state before collecting data [105]. All these restrictions are making outdoor and mobile usage of EEG very limited: any movement of a user will create recording artifacts and every electronic device in the vicinity will affect the recorded electromagnetic field.

\subsection{Electrooculography}

EOG is a non-invasive technique for estimating the permanent electric potential difference between the cornea and ocular fundus of the human eye as shown in Figure 9 [123]. The eye can be represented as an electric dipole with a positive pole at the cornea and negative on the retina. Change in the direction of gaze, controlled by eye muscles, transforms the configuration of the dipole, resulting in EOG potential. The detectable changes of the potential in the range from 0.1 


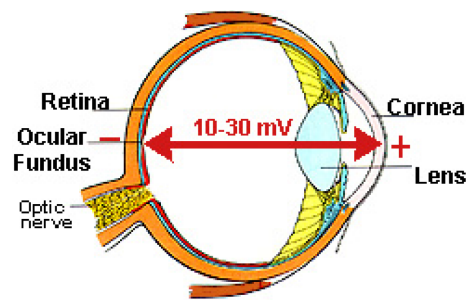

Fig. 9. Electric potential measured by EOG (from Reference [123]).

to $2 \mathrm{mV}$ within $500-\mathrm{ms}$ time interval, on the frequency of $0-30 \mathrm{~Hz}$, and resolution of one degree can be detected by electrodes placed on forehead, temples, and upper side of the cheek [53]. It is assumed that the relation between the generated potential and eye movement is linear [215], however, due to the fact that tissues around the eye are not distributed uniformly, the propagated signal measured on the surface can be considered only as an approximation of the biological reality [123]. Besides, the EOG signal is influenced by the noise generated between the electrode and the skin, myoelectric activity of facial and eye muscles, and metabolic state of the tissue; potentials generated by the vertical eye movements are also sensitive to movements of the eyelids.

\subsection{Muscular Myoelectric Activity}

Motor intentions, independently of their motivation (e.g., speech articulation, balancing, or intended gestures), emerge in the Motor area of the human brain. The control signal goes through the submotor cortex, spinal cord, and peripheral nervous system toward a motor neuron innervating a muscle. Nerve impulse that is being conducted through neuromuscular junction triggers the release of neurotransmitters into the synapse of motor neurons. The consecutive propagation of action potential causes the flow of ions, that generate time-varying myoelectric potentials. As a result of the described process, target muscle contracts in a desired fashion [111]. Elicited myoelectric patterns can be observed using EMG invasively via electrodes incut directly into muscles, or non-invasively using surface electrodes. sEMG uses electrodes placed on top of specific muscle or electrodes organized as a grid [192]. Signals are measured as a difference between recording electrode(s) and ground (or reference) electrode or utilizing several active electrodes, as in many commercial EMG devices [33, 41]. Similar to the EEG, the recorded sEMG signal is a composition of potentials from several neurons, also smeared by the volume conduction through tissues, skin, and skin-electrode interface.

2.3.1 Myoelectric Aspects of Speech. Speech, as a process, can be considered as a transformation of chemical neuronal activity into acoustic signals via several stages: Brain activity inside Broca's area triggers electrochemical impulses in the peripheral nervous system that, in turn, controls jaw, lips, tongue, larynx, and others to produce mechanical vibrations of certain frequencies causing acoustic waves. It is interesting to note that myoelectric signals in ortofacial muscles appear approximately $60 \mathrm{~ms}$ before the actual articulation [192]. Whispered, murmured, and normal speech is a process that results in vocal vibrations. Silent Speech, however, is a process when articulating muscles (see Figure 10) are operated the same as in case of normal speech, but the respiratory activity is suppressed and no sound is produced. The electrical activity of ortofacial muscles related to the process of silent speech can be captured using sEMG electrodes. The silent speech interaction paradigm might not provide sufficient privacy as it exposes visual muscular articulation so that an adversary can decode it. 


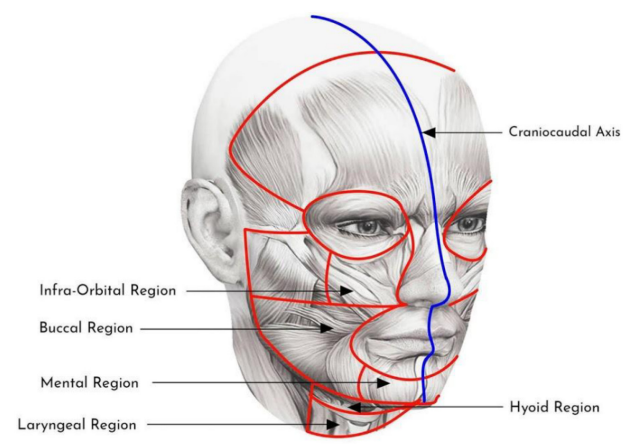

Fig. 10. Muscle regions involved into speech production (from Reference [111]).

\section{EXG HARDWARE}

In this section we discuss NUI hardware solutions offered by industry and academia. First, we introduce the mechanics of electrodes, their types, and applications. Then we provide an overview of portable EEG solutions, EMG, and EOG hardware. We mention several hardware open source platforms suitable for research or non-Industry deployment and usage. We consider only wireless devices that do not require wired connections for power or data transmission. Most of them can last for several hours autonomously, for example, number 18 from Table 7 can be even charged from a power-bank while recording. The target activity of these devices is to acquire biological signals in mobile scenarios or combined with XR hardware while not restricting user's mobility.

We also show the API those devices expose to users, developers, or researchers. We consider the number of channels $(N)$, the temporal resolution of each channel (TS, in $\mathrm{Hz}$ ), spatial resolution ( $S R$ in bits), and the transmission protocol, e.g., Low Energy Bluetooth. Thus the effective bandwidth $\left(B_{E}\right.$, in bits $\left./ s\right)$ of the utilized communication channel can be represented as follows:

$$
B_{E}=N \cdot S R \cdot T R \text {. }
$$

The effective bandwidth influences the choice of transmission protocols, the requirement for channels to offload computations related to bio-signal processing. Classification software and algorithms that are intended to use in real-time should be capable of processing the data frames formed by hardware or employ downsampling techniques. It is worth noting that, regarding the sampling frequency of presented devices, according to the Sampling Theorem, when sampling the signal of frequency $f$, the sampling frequency should be twice as high, i.e., $2 \cdot f$. Thus, for example, devices whose sampling rate is $256 \mathrm{~Hz}$ can reliably capture oscillations on frequencies $\leq 128 \mathrm{~Hz}$.

\subsection{Electrodes}

$E x G$ is a shorthand for EEG, EMG, electrocardiogram (ECG), and EOG [44]. Electroglottography, which is a non-invasive technique of monitoring vocal fold vibration by sensing the electrical conductance between two electrodes placed on the neck, can be considered as one of the representatives of ExG as well. There are multiple types of ExG electrodes, as depicted in Figure 11, that can be classified by the following criteria:

By Contact Type. Wet electrodes require the application of conductive gel or paste between the surface of the scalp and electrode to eliminate obstacles for charge propagation. Such electrodes can't be considered for everyday usage in consumer-grade hardware: Gel or paste turns greasy, and deployment type takes up to one hour. Wet electrodes provide high signal quality and are used in medical-grade hardware [182]. Dry electrodes require no conductive substance, electrodes 


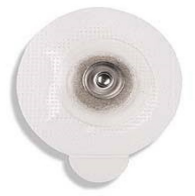

(a) Wet

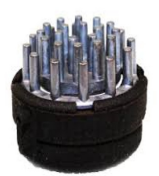

(b) Dry, metal pins

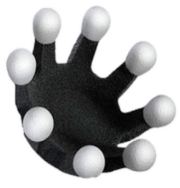

(c) Dry, hair

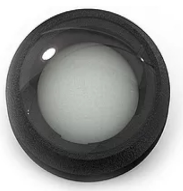

(d) Dry, skin

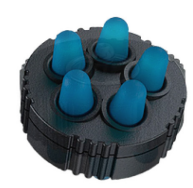

(e) Dry, universal

Fig. 11. Examples of different types of electrodes.

are contacting with skin or hair directly. The air space is minimized by the pins on the end of electrodes. Some dry electrodes (e.g., OpenBCI, number 14 in Table 6) contain springs pushing the pins toward the scalp. Dry electrodes might cause discomfort and irritation, continuous usage is questionable [182]. Another type of electrodes is semi-dry: Their Tips can slowly release a tiny amount of electrolyte liquid to the scalp. Emotiv Insight 5 (number 2 in the Table 5) is one of a few examples of hardware with semi-dry electrodes. However, refilling the syringe inside every electrode can be also considered as an inconvenience, that affects acceptance by users.

By Applicable Surfaces. To provide a better signal in various conditions different types of electrodes are used. A set of pins on the end of electrodes is used for the acquisition of the signal through hair; skin electrodes usually have a plain surface; while universal electrodes can provide a decent quality of records from any surface.

By Amplification Capabilities. Active electrodes contain an amplifier with factor of 1-10 to minimize the interference, while passive electrodes do not contain such an amplifier on-site.

By Material. Usually electrodes are made from gold $(\mathrm{Au})$ or silver $(\mathrm{Ag} / \mathrm{AgC})$. Gold electrodes are suitable for measuring different modalities (e.g., EMG, ECG), while silver ones proved to be more reliable to record EEG signal of low (below $1 \mathrm{~Hz}$ ) frequencies [182].

\subsection{Brain-Computer Interfaces}

Tables 5, 6, 7 and 8*, in Appendices A, B, and C list up-to-date portable EEG headsets. Several criteria might be introduced to define portability and user-friendliness; we adopt metrics from Reference [69]:

Ease of Deployment. Preferably, dry or (semi)dry electrodes should be used, enabling a deployment within a reasonable amount of time $(<5 \mathrm{~min})$. Ease of deployment also implies the speed and required qualification efforts to obtain, record, and analyze a signal from a recording device.

Portability. The EEG hardware should be capable of producing signal good enough for betterthan-random guess analysis in non-shielded mobile scenarios in naturalistic settings with a lot of physical and electromagnetic noise.

API Availability/General Applicability. Classical medical EEG headset system comes with proprietary highly-integrated hardware and software environment, that provide a fixed list of certain analysis methods. A device should expose API with access to raw data. For example, EMOTIV headset (number 2 in Table 5) requires a paid subscription to access raw EEG data and still limits the number of recordings per day.

\footnotetext{
${ }^{*}$ In the presented tables we used several simplifications: $\rightarrow$ is used to depict the digital downsampling performed on board. For example, $1,024 \rightarrow 128$ implies that the signal is recorded at $1,024 \mathrm{~Hz}$, but the digital data stream contains 128 samples per second. OR is used to describe the various capabilities of a single device: $500 \mathrm{~Hz}$ OR 1,000 Hz means that a device can capture signal on both frequencies, $500 \mathrm{~Hz}$ and 1,000 Hz. / is used to combine several products within a certain product line with similar characteristics. For example, Cognionics QUICK (number 10 in Table 6) product line consists of three similar products, differing only in electrodes count.
} 
In addition to EEG acquisition devices presented in Tables 5, 6, and 7 there exist several DIY EEG projects [27, 170]. For example, ModularEEG [170] is a community of enthusiasts that aim to build low-cost EEG acquisition hardware and build a software ecosystem around it assisting signal collection and classification. A hardware device, based on this platform, EEG-SMT [160] has been used by Angrisani et al. [48] to utilize SSVEP-interface in AR for monitoring and inspections in the industry. Another example of a configurable hardware platform for bio-signal acquisition is BITalino [65]. BITalino offers several sets of hardware for collecting EEG, EMG, ECG signals, as well as amplifiers and connectivity (Bluetooth or BLE) boards.

Several studies accessed and compared the capabilities of consumer-grade EEG devices. Ratti et al. [184] compared the performance in terms of power spectra similarity of B-Alert X24 (number 17 in Table 7) and Enobio 20 (precise medical-grade EEG headset) with consumer-oriented Muse and NeuroSky Mindwave (number 6 and number 5 in Table 5, respectively). This study showed that Neurosky EEG obtains signals from electrode location Fp1 of similar power spectrum with medical-grade hardware, while increased values of power spectra were detected in signal collected by Muse headset. Muse demonstrated the highest relative variation across multiple acquisitions; both consumer headsets suffered greatly from artifacts such as eye blinks. Ratti et al. conclude that although EEG signal can be successfully collected by all devices, medical-grade hardware offers better quality and more reliable signal suitable for many applications.

In another study, Raduntz et al. [181] compared EPOC (number 1 in Table 5), Mindo Trilobite/Jellyfish (number 20 in Table 7), BR8PLUS (number 19 in Table 7) and g.SAHARA/g.LADYbird (electrode layouts for g.Nautilus, number 23 in Table 7) employing 24 participants performing multiple BCI-related tasks for 6 consecutive days. Devices were compared based on multiple criteria: Signal-to-Noise ratio (SNR), the ratio of the power of the meaningful signal and the power of the unwanted signals, in decibels; the proportion of recorded artifacts; and quality of the frequency domain. The study concludes that mobile systems with wet electrodes are producing more stable signals of better quality than the systems with dry electrodes. Nevertheless, the quality of recorded signal using dry electrodes EEG was characterized by the authors as "comparable and promising." Signal quality and research applicability of Emotiv Epoc+ (number 1 in Table 5) is accessed in Reference [69]. Raduntz et al. outline that despite several challenges, consumer-grade EEG headsets represent a useful addition to BCI research. In Reference [135] overview of research projects in the area of $\mathrm{BCI}$ hardware is presented; Lin et al. conclude that existing $\mathrm{BCI}$ hardware is held back by many factors and they expect more emerging projects in this area tackling today's challenges. Thus, the scope of BCI paradigms that are applicable in mobile and XR applications is limited; performance accuracy, as well as intersession stability, is significantly inferior to professional imaging devices [184].

\subsection{Surface Electromyography}

The mapping of the collected myoelectric signals to the user's gesture is complex and highly dependent on the quality of the collected signals and the selected classification method. sEMG devices are able to collect and amplify the signals generated by the human muscles and, depending on their processing and networking capabilities, process and transmit them to other devices. There exist a wide variety of sEMG recording hardware aimed for medical purposes: Solutions from BTS Bioengineering [67], MotionLabs [159], Otto myoblocks [1]. PicoEMG [201] sensors can be organized as a mesh over muscles of interest; each sensor is capable of sampling data at 2,000 $\mathrm{Hz}$ for up to 12 hours, weighing only 7 grams.

Overview of commercial consumer-grade EMG devices is presented in Table 9. The first entry, Myo band by Thalmic Labs, is one of the most popular sEMG recording devices used in many HCI studies: It is more affordable than medical-grade sEMG acquisition hardware; dry electrodes and 


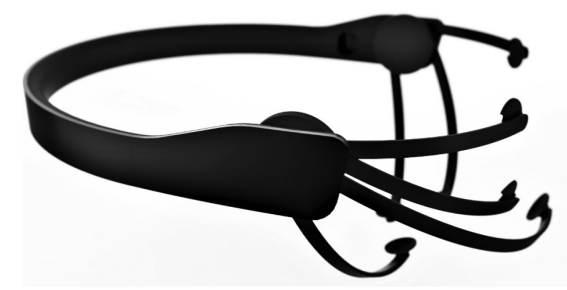

Fig. 12. SSI mobile wearable device [111].

band form factor ensure high SNR of the collected signal and quick deployment; access to raw data and BLE connectivity provide easy integration with mobile and XR applications out of the box.

Côté-Allard et al. [75] built 3DC Armband, a low-cost 3D-printed custom sEMG band employing 10 electrodes at $1,000 \mathrm{~Hz}$, and in-house designed PCB. 3DC Armband outperforms the Myo band in terms of SNR and accuracy of recognized gestures. All blueprints and related source codes of the 3DC band are made available to the public. Another open source project, MyoWare Muscle Sensor board [99], is an Arduino board with two electrodes (and a reference one). It captures, rectifies, and filters EMG signal, however, power supply and data transmission components should be designed and built separately. Open source solutions offer the flexibility of deployment and integration with XR applications and hardware, while manufacturing and assembly require additional expertise.

To obtain myoelectric signals related to speech production, sEMG electrodes are placed in strategic locations on the face and neck: Wand et al. [210] employed five sEMG electrodes sampling at $600 \mathrm{~Hz}$; Deng et al. [77] used eight wireless sEMG sensors that are a customized version of the Trigno wireless sensors [101] sampling at $20 \mathrm{kHz}$; in multi-modal approach to SSI, as described in Reference [88], besides using Microsoft Kinect Depth camera, a custom-built ultrasonic sensing device, Freitas et al. used five pairs of sEMG electrodes from Plux [189] capable of sampling the signal at $500 \mathrm{~Hz}$. Several design aspects apply to a mobile SSI acquisition device to obtain a robust sEMG signal for silent speech processing. First, electrodes should not change their position during various activities, e.g., walking or running, to maintain consistency of recorded signal within one session. Another consideration is that electrodes should be deployed in the same position every time device is put on to maintain the consistency across multiple sessions. Additionally, positions of electrodes should be adjustable to be placed on top of the same muscles independently of facial shapes of multiple users. A device meeting these requirements [111] is shown in Figure 12: a wearable fixed around the top part of the neck with electrodes placed on the ends of adjustable manipulators that in the same time provide desirable rigidity for steady signal acquisition. The described device uses 7 wet golden plated silver or passive dry $\mathrm{Ag} / \mathrm{AgCl}$ electrodes; sEMG signals are sampled on $250 \mathrm{~Hz}$. A fusion of a silent speech device with a self-contained breathing apparatus is presented in Reference [110]. Such a system employs two wet EMG electrodes placed under the chin and on the left side of the neck. This work shows the compatibility of SSI with specialized equipment in cases where silent speech communication is most applicable and useful-noisy and harsh environments.

\subsection{Electrooculography}

EOG potentials can be recorded by electrodes placed on both temples, upper cheek, and forehead. It also claimed that retino-ocular potentials are best detected on the lids and external canthi (the bone on the side of the eye) [123]; however, such a placement might be considered obstructive and irritative, limiting the range of eye movements. In some studies EEG hardware with electrodes placed on specified positions around eye is used to measure EOG potentials: g.USBamp (number 


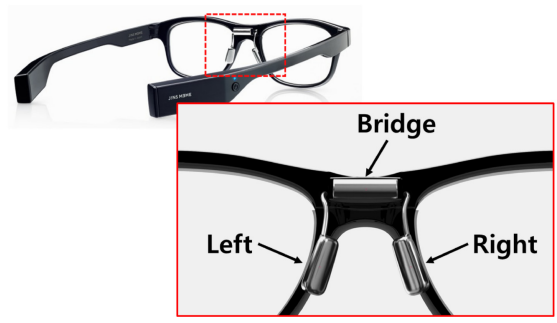

Fig. 13. Electrodes placement of commercial EOG acquisition device.

23, Table 7) in Reference [144], OpenBCI (number 14, Table 6) in Reference [204], or medical-grade Mobi8 by TMSI [206] in Reference [200]. Many researchers have designed and built customised EOG-signal acquisition solutions: Reference [53] employed wet electrodes and custom made amplifier; hardware described in Reference [145] consists of two electrodes and Arduino-based amplifier; and $\mathrm{Wu}$ et al. [215] used five wet electrodes, in-house designed amplification hardware with Bluetooth module.

Bulling et al. [68] developed EOG glasses with five dry electrodes capable of detecting eight different eye gestures with a built-in battery for up to 7 hours of autonomous functioning. Similar glasses are presented in Reference [72], employing three dry $\mathrm{Ag}-\mathrm{AgCl}$ electrodes, a custom-made amplifier, signal conditioning unit, and wired connection to a PC for signal processing. Another proof-of-concept prototype is the AttentivU glasses by Kosmyna et al. [118]. AttentivU glasses are designed as a socially acceptable pair of glasses with embedded dry silver EOG electrodes (two nose pads and the nose bridge as reference) and two EEG electrodes (TP9 and TP10 according to the 10-20 EEG positioning system) on the temple tips of the glass frame. Captured EOG and EEG signals can be processed in real-time by an on-board processor or sent to an external processing device via Bluetooth. Kosmyna et al. provide a comparison of signals recorded with AttentivU and wet electrodes, concluding that obtained EOG and EEG signals can be used in multiple activitytracking applications, such as attention monitoring.

There are commercial EOG products on the market, such as Jins MEME ES [153], that uses three dry electrodes (as depicted in Figure 13) with an additional six-axis IMU. These glasses are reviewed in Reference [103] by Ishimaru et al., where the authors present the methods of distinguishing several everyday activities based on sensors data, such as typing, eating, reading, and talking. Another example of commercial EOG glasses is Imec Eye-tracking glasses [102]. It is mentioned that the sampling rate of $256 \mathrm{~Hz}$ is superior to any video-based eye-tracking system, as well as energy and cost efficiency of such a solution surpasses video-based systems. These glasses are equipped with a battery allowing them to last up to 10 hours and a Bluetooth module for wireless communication.

\subsection{ExG Hardware Challenges}

Although several studies evaluate the capabilities of modern consumer-grade signal acquisition hardware $[69,181]$, there exist multiple challenges in the filed of bio-signal imagining modalities.

Temporal Limitations. Even though sampling frequencies of several EEG and EMG devices are high, instant voltage drops (for example those related to cognition) might not be detected by the acquisition hardware.

Spatial Limitations. The density of the electrodes is limited by the obvious physical constraint on the head, arm, or face. However for some applications, like precise gesture recognition, it is better to have dense electrode arrays over certain muscles [157]. 
Signal-to-Noise Ratio. Any mobile device is susceptible to internal and external noise. Leveraging the effect of electromagnetic interference and movement artifacts both programmatically and on the hardware level is an important milestone on the way of delivering reliable and robust mobile imagining modalities.

Energy Efficiency. Management of a power supply for acquisition hardware and bio-signal processing facilities is a crucial task in deploying user-friendly NUIs. The capacity of batteries that users can carry is physically limited, so all wearable devices will compete for power supply. Energyefficient offloading of computationally heavy tasks may reduce the need for power [196, 227].

Signal Transmission. Wearable devices and sensors are additionally constrained by networking interfaces. Delays, interference, collisions, and protocol bottlenecks are challenges to address [226].

Users Comfort. In the case of user comfort several problems arise: The whole system can be bulky or obtrusive or connected electrodes might cause discomfort. Dry electrodes are causing pain and irritation, which makes them not suitable for long usage; while conductive gel used in wet electrodes leave sticky stains and requires reapplication in certain cases.

\subsection{Hardware Challenges of Combining XR and ExG Hardware}

It is clear that BCI, EOG, or SSI electrodes will compete for the space on the user's head and/or face with AR glassed, HMDs, and other output devices. Usage of head-mounted MAR systems, like Hololens, can block placement of electrodes in frontal and temporal lobes, while electrodes in central areas of the scalp can be placed freely. In some cases, electrodes can be placed around the display of XR HMD's chassis, but it will most likely result in discomfort or recording artifacts and signal interference [180]. Systems like DSI-VR300 (Number 15 in the Table 6) and Looxid VR (Number 16 in the Table 6) are examples of how this issue can be addressed. Yet, this tight integration comes with its own shortcomings: the amount and configuration of electrodes are fixed (limiting the potential BCI scenarios); ExG electrodes, as well as MAR or VR headset, cannot be used separately; vendors can potentially limit the scope of compatible software, SDKs, AR/VR platforms, and so on.

Another challenge is ergonomics in general. A combination of complex hardware can be bulky and require longer deployment time, MAR headsets, for example, and ExG recording hardware can strain the user's neck and head negatively affecting the user's experience. Requirements for total weight and comfort might restrict the allowed weight and, as a consequence, capacities of batteries that can be placed on the user's head, thus creating another competition for the power supply.

Moreover, Ke et al. [113] have shown that there exists a gap between the performance of visualbased BCI (SSVEPs) on traditional computer screens and OST AR specifically. The performance difference is mainly derived from limited display refresh rates, color degradation and blurriness, and distraction caused by real-world objects that partially overlay SSVEPs markers. All these factors affect the perception of visual cues; meanwhile, the necessity to focus on a semi-transparent screen leads to additional fatigue.

PhysioHMD [64], a software and hardware platform for collecting and analyzing physiological signals from HMDs users, addresses some of the mentioned challenges. A hardware implementation of PhysioHMD is a flexible printed circuit board with a plethora of ExG electrodes (ECG, Electrodermal Activity, sEMG, EOG, and EEG), shaped as an eyemask, takes advantage of close contact of an HMD and face (see Figure 14). Placement of the electrodes, for example, under the foam of a VR headset, introduces affective computing (such as recognition of emotions and facial expressions) into XR applications. Meanwhile, the software framework is open source and contains multiple integration tools. Such an approach to integrate XR and ExG, however, still limits 


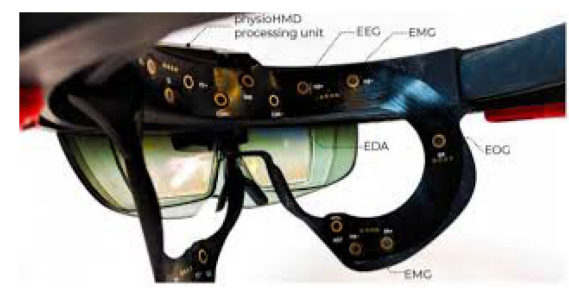

Fig. 14. PhysioHMD [64].

the scope of applicable BCI scenarios; electrodes placed between the user's face and a headset can cause additional irritation; electric interference and motion artifacts from the headset itself reduce SNR of the obtained ExG signals.

\section{EXG-BASED APPLICATIONS IN THE CONTEXT OF XR}

In this section, we outline five major vectors of application of ExG technologies in XR: (i) inconspicuous interactions, interactions with (ii) virtual objects in XR and with (iii) real-world objects through XR, (iv) affective computing, and (v) biometrics and security. In this section, we illustrate how interactions in XR can benefit from ExG-based inputs in each of the identified directions on the application level.

Inconspicuous interactions. ExG-based interfaces can provide ways to interact with XR content in a concealed, non-obtrusive, non-disturbing (from the perspective of bystanders) way. In terms of MAR, performing gestures in public spaces attracts unwanted attention and can be physically constrained [149]. Similarly, voice inputs or direct interaction with input devices in several social contexts, for example, classroom or library, are inappropriate [77]. Interaction techniques that are more socially acceptable and less physically and mentally demanding contribute toward wide adoption and better usability of XR.

In that context, $\mathrm{BCI}$ is an ultimate modality for the inconspicuous interaction approaches as thought-based input does not require space to perform control gestures or a touch-surface and is imperceptible for bystanders. BCI can be used for controlling devices hands-free, silently, and without disturbing other inhabitants of the environment, or when other modalities are not available [47, 229]. In the motor imagery endogenous control paradigm, when a user thinks of action, instead of performing it, is an exemplary subtle input, suffering, however, from low ITRs (up to $23 \mathrm{bpm}$ in Reference [222], where MI is used to control a robotic arm with visual feedback in OST $\mathrm{AR}$ ). As an alternative to the gaze or head pointing on XR HMDs, four-directional motor imagery is applied to the cursor control [124]. In practice, most BCI paradigms of direct control rely on gaze and visual attention to a set of markers.

Motivated by the two-way interaction between humans and the digital overlays projected through MAR hardware, gaze pointing including IMU-driven head pointing and eye pointing has drawn attention to the design of mobile headsets. For example, Microsoft Hololens employs head pointing to target a user-interested item, and subsequently, a touch-based clicker enables users to confirm the target selection. Although it is preferred by users [219], head pointing is not ergonomic for long-term usage. Alternatively, eye pointing achieves more subtle movements than the head pointing, which also serves as an alternative solution for pointing and selecting an attentive target after a predefined duration of time (i.e., dwell time) [174]. However, the key challenge of eye pointing is to find the convergences for accurate and precise timing of Gaze input [114], due to the Midas Problem [207]. Head pointing and eye pointing can work together and compensate for the weaknesses of each other: Eye pointing alone is faster than head pointing, but head 
Table 1. EOG ITRs

\begin{tabular}{rccc}
\hline & Cmds & Acc. & ITR \\
\hline Tamaki et al. [204] & 2 & $94 \%$ & $22 \mathrm{bpm}$ \\
\hline Wu et al. [215] & 8 & $89 \%$ & $35 \mathrm{bpm}$ \\
\hline Atique et al. [53] & 5 & $80 \%$ & $57 \mathrm{bpm}$ \\
\hline
\end{tabular}

Table 2. EMG ITRs

\begin{tabular}{rccc}
\hline & Cmds & Acc. & ITR \\
\hline Moin et al. [157] & 21 & $98 \%$ & $837 \mathrm{bpm}$ \\
\hline Shatilov et al. [196] & 11 & $92 \%$ & $669 \mathrm{bpm}$ \\
\hline Côté-Allard et al. [75] & 11 & $89 \%$ & $1567 \mathrm{BPM}$ \\
\hline
\end{tabular}

pointing allows greater targeting accuracy [122]. Therefore, existing works propose the mix of them to improve the usability mobile headsets [209]. The recent advancement of eye pointing goes toward the Midas-free approach, which sheds light on sole eye pointing for input modals on mobile headsets [45]. For example, WorldGaze [152] emphasizes the user's gaze on the object in physical reality. That enables voice assistants to identify specific objects in the respective environmental context, leading to more intuitive conversations between a human user and a voice assistant (i.e., this/that/there, instead of specifying the full name of the items and locations). In the case of hand-held AR, WorldGaze relies on the computer vision estimation of head gaze ray from the frontal camera of a smartphone and projects it into the 3D scene captured by the rear camera.

Given the XR-aware ExG hardware solutions (e.g., Attentive UI glasses [118]) or methods of incorporating ExG electrodes into XR headsets (e.g., PhysioHMD [64]), EOG augments the set of available gaze tracking modalities for head-mounted displays. In its current state (see Table 1) EOG can serve as a complementary modality for gaze tracking, addressing the Midas problem by differentiating between involuntary eye movements, saccades, and intentional eye globe rotations [208]. As a part of evaluation of EOG glasses (see Figure 2(b)), Bulling et al. [68] designed a computer game, where users were guided to repeatedly perform ocular gestures (e.g., diagonal or horizontal movements). During the experiment, there was no restriction on the movement of the head and upper body. As a result, around one-third of all the participants reported that they had difficulties concentrating on the eye gesture input task. This study illustrates the feasibility of the application of EOG for XR input (authors present a combination of the proposed glasses with OST AR and a wearable keyboard), simultaneously outlining its limitations, i.e., eye fatigue and relatively low ITR.

Information transfer rates of sEMG-based interfaces, however, are significantly higher compared to EOG, presented in Table 2. Besides, sEMG acquisition hardware does not compete with XR headsets for a space on the user's head or face, while also leaving the user's hand unoccupied. Continuous interplay with physical objects during interaction with sEMG-based systems [195] blurs the boundary between subtle interfaces of XR and physical realities. Arguably, control gestures still attract the attention of bystanders, but sEMG-based recognition does not require lifting hands to eye level, allowing more concealed input than the computer vision-based approach. To not overcomplicate the interaction protocol and exploit users' familiarity with existing input systems, sEMG is combined with auxiliary modalities, such as IMU, to build XR interfaces [121]. 
Last, sEMG-based SSI delivers a discreet method of free-hand interaction with computing systems, including XR interfaces. Easier deployment of dry electrodes over the ortofacial muscles and less constraining, more comfortable hardware [111] are the benefits of SSI, compared to EEGand EOG-based interfaces. Non-demanding, mentally and physically, SSI allows interacting with extended realities in a less obtrusive manner, additionally allowing robust voice communication in noisy environments. Voice, distorted by external noises, masks, or garments, can be reliably captured by SSI. An example of SSI assisting the firefighting process is presented by Jorgensen and Dusan [110], where EMG electrodes are embedded into breathing apparatuses. As a part of the two-way interaction paradigm, OST AR can provide feedback and display vital information. Furthermore, once the vocabulary of SSI is large enough and means of output are provided (e.g., bone-conducting headphones), networks of people can exchange information via SSI. Such systems can be deployed in environments where noises are prohibited or socially unacceptable [77].

Interaction with virtual-world objects in XR. XR headsets create a scenario where the digital contents are not directly reachable due to the missing of keyboard and mouse duos. The most recent works on interacting virtual-world objects in XR show the formidable hurdles, regardless of the robust and widely employed sensors, such as tangible touch interfaces or camera-based hand gestural inputs. As reported in a recent work, named UbiPoint [130], sole mid-air pointing on keyboards can reach only 0.99 Words-per-Minutes (WPM), $\sim 5$ successful key clicks on a keyboard in one minute, while the mixed design of mid-air pointing and touch-based interfaces achieves a fourtime faster WPM (3.99 WPM, 20 successful key clicks per minute). However, the performance of pointing and targeting on virtual items, resemble mouse pointers on desktop computers, are significantly slower than the smartphone counterpart (i.e., >38 WPM [172]). In the past decade, numerous studies focus on the manipulation of virtual objects using hand gestures [131]. Recent efforts of interaction design of XR move toward the morphology of hand gestures (e.g., gripping of virtual objects) and the investigation of more realistic feedback that connects to the real-world environments (e.g., requiring muscle strength to roll a virtual wheel in VR [187]).

According to the interaction design theory of the Interaction-Attention Continuum [56], the hand gestural interaction can be regarded as the class of focused interaction that demands for conscious intentional, and direct precious control, e.g., controlling users' hands in particular gestures, and displacement of the hand grasp from one position to another. In contrast, the adjacent and connected class of peripheral interaction (in a relationship of Venn diagram), requiring subconscious intentional, and direct imprecious control. We consider the EXG technologies to leverage the subconscious signals inside the users' bodies, enabling the interaction of virtual objects in XR in a less precious way than the above examples of hand gestural controls.

When the continuum of digital and physical reality becomes blurry with the immersive counterparts, the physical reality becomes the indispensable portion of the HCI interfaces [106]. For example, buttons or icons are regarded as the most ubiquitous interfaces in both the physical and digital artifacts [108]. BCI serves as an alternative channel for us to interact with the artifacts at the blurred boundary. Coogan and $\mathrm{He}$ [73] deploy BCI2000 native application in the Unity 3D environments, which demonstrates the feasibility of interacting with the enriched objects in immersive reality, including 2D GUIs (e.g., icons, windows, and menus), as well as 3D objects resembling our daily life.

Virtual keyboards, a vital aspect of interaction with digital worlds, can be built upon ExG modalities. BCI-driven keyboards, also referred to as BCI-spellers, suffer from low input bandwidth [186]. The state-of-the-art high-speed speller [162], based on SSVEPs, reaches 50 bpm by presenting to user 32 flickers of 8 different frequencies and 4 phases. To demonstrate the viability of miniature aVEPs paradigm, that is more suitable for XR environments, $\mathrm{Xu}$ et al. [217] built 
a 32-alphanumerical speller, achieving lower ITR (30 bpm). Virtual keyboards can be also built upon multi-modal ExG interfaces. For example, a combination of EMG input and SSVEPs paradigm showed high accuracy of $99.2 \%$ with ITR (50 bpm) [165]. Gesture input keyboards [35, 121], however, utilize both arms and significantly increase the efficacy of text input.

sEMG provides an alternative interaction experience within XR environments. XR interfaces, that are less convenient for direct touches such as the ones on smartwatches and smartglasses [120], can be manipulated by IMU-driven forearm movements and sEMG-based muscle controls. Haqe et al. [92] proposed a system of controlling any personal computing device utilizing the IMU embedded into Myo Band to control a cursor and inferred gesture to input different commands, providing an alternative to mice. The strength of muscle contraction (as deducted from an sEMG signal) can serve as an input modality as well. For example, users can tense their forearm muscles to support the intended interaction with virtual objects such as pushing, rotating, and lifting virtual objects by varying the amount of applied force [187]. As a result, users achieve higher enjoyment and immersion with the digital contents.

Finally, EEG-based interfaces offer a way of subconscious labelling of digital content, unavailable though classic I/O interfaces [76]. Abuhasira et al. [143] used brain generated labels to train an image recognition network. Images at the rate of $4 \mathrm{~Hz}$ were shown to a patient (wearing DSI-24 EEG recording device (Number 21 in Table 4)). Obtained EEG signal is then used to generate socalled soft labels representing the confidence of a certain class. Finally, these labels along with initial pictures are fed into an image recognition network for training. For 2-labels classification, this work outperforms the network trained on the manually labelled pictures (AUC 0.91 vs. 0.89) within much less time required for labelling (22.8 minutes vs. 257.8 minutes).

Interaction with real-world objects through $X R$. On the continuum of extended realities, augmented and mixed reality technologies pivot on the interplay with real-world objects and environments. AR, for example, allows users to identify objects [125], display hints and contextual information [61], orchestrate smart home appliances [229], and so on. ExG-based NUIs can provide hands-free input, augmenting traditional ways of interaction with physical objects, e.g., natural control (by gestures or motor imagery) of robotic manipulators and robots [168]. In turn, the twoway interaction benefits from AR feedback: Zeng et al. [222] have shown that the overall number of issued control commands to achieve a desired position of the robotic arm was lower in the conducted experiments provided the feedback.

As an example, smart garments can be controlled by BCI [154]. In that case, BCI serves as a sensory device to detect the users' mental state and adjust the appearance of the smart garment. In a similar way, BCI-driven sensory input affects the state of a gadget in the interaction loop: objects are identified, addressed through XR and transformed accordingly; the subsequent change of the state is displayed by the XR interface [229]. Si-Mohammed et al. [197], have proven the feasibility of combining AR and BCI: The authors explored different positions of SSVEPs markers regarding the controlled physical object in AR and applied their solution to control a mobile robot.

sEMG is mostly used for natural dexterous control of prosthetic devices [196]. The grand challenge of sEMG-based gesture recognition is to increase the number and precision of recognized hand postures to reflect the real sensorimotor interaction with the physical world (for example, 21 classified hand gestures by utilizing a compact mobile high-density EMG grid in Reference [157]). Similarly, control of robotic devices is achieved with multi-modal ExG interfaces: Zhang et al. [225] combined EOG, EEG, and EMG to operate a robotic soft hand; Tayeb et al. [205] manipulated robotic hand simultaneously with sEMG and 2-label MI. Although these works are not directly applied to XR, sEMG and multi-modal interfaces can be utilized to build more effective XR input interfaces (compared to SSVEPs BCI [48]) and visual feedback, in turn, can improve the speed and precision of the manipulation [222]. 


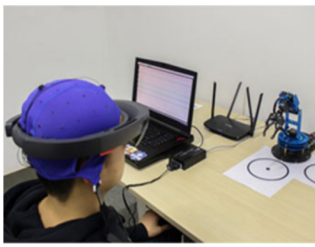

(a) EEG for robotic arm (b) control in AR [113]

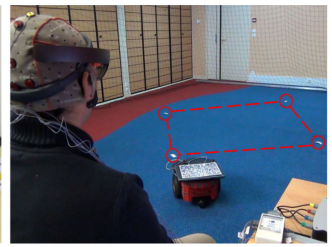

(b) EEG for drone control in AR [197]
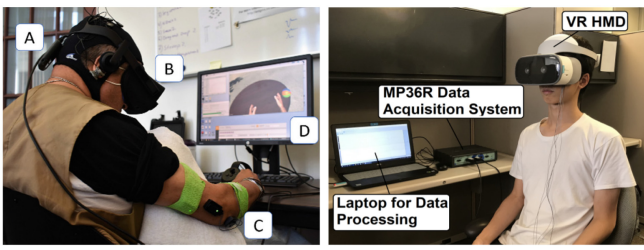

(d) EOG Authentication in VR [142]

Fig. 15. Examples of ExG applications in XR.

Augmenting the interactions. ExG-based interfaces bring additional input channel-physiological and -neurological information, mostly unavailable to be captured by traditional input devices. ExG imagining techniques can effectively monitor mood, fatigue, attention, and so on. Consecutively, virtual interfaces and environments in XR can adapt accordingly to reflect the observed mental state or to induce a desired cognitive status (e.g., increase a driver's attention [145]).

According to Viveros et al. [55], the analysis of EEG signals can reveal a user's preference, perception, and attitude toward certain types of interfaces: colors, fonts, text size, size of the icons, the position of the elements, and other characteristics of interest. Given the reliable measurements of a user's anxiety, enjoyment, or satisfaction, novel interfaces and experiences in XR can be shaped toward better usability. EEG signals can serve as an input to handcraft immersive and adaptive virtual worlds as well. Potts et al. [178] proposed a neuro-feedback driven AR application for kinetic meditation. In their study, the intensity of alpha-band is analyzed to estimate users' mental state. The monitoring of mental state gives significant insights to game designers about the users' emotions [98] as well as the user experiences [80] throughout the gaming process. AR scene reflects the change in mental state and surroundings are being adjusted accordingly in virtual reality. Such an application of neuro-feedback increases user engagement in XR applications [179]. In addition, brain activity reveals the comprehension of visual or vocal stimuli, such as second-language. BrainCoDe [191] leverages EEG to analyze the user's understanding of a second language vocabulary during text reading on-screen or listening to audio content. When the user with immersive headsets does not understand the contents or objects, the BCI interface provides feedback to translate the uncomprehended words and phrases. When the blurred interfaces have inclined to our reality [106], such tools for monitoring our levels of comprehension of unfamiliar texts could be extended to physical counterparts and make users more aware of the environment [214]) improving cognitive abilities [66], as many existing works propose augmented information on texts [50], daily objects [199], and office environments [97].

Apart from a mental state, series of images corresponding to the user's vision can be partially inferred from the brain activity. A deep neural network is capable of reconstructing colored discernible silhouettes based on captured neuronal activity of the visual cortex [183]. Such a transformation can potentially enable XR systems to be more aware of the user's environment contexts in a privacy-preserving fashion (from the perspective of bystanders).

sEMG interfaces can augment interactions with virtual worlds by providing contextual information about the interaction itself. Becker et al. [59], for example, proposed an approach to identify the exact finger being used for interacting with touch devices (or any surface) and to measure the force applied. Among applications of this solution, Becker et al. mention the possibility of turning any surface into a touch-enabled input device and advanced text marking. Furthermore, sEMGbased interaction enables the detection of the user's muscle status and thus enrich the gestural inputs acted on our daily objects such as pen [151]. That is, the augmented daily objects can serve as an alternative channel for user interaction with XR applications. 
EOG is commonly applied as an affective state interface: multiple studies [145, 231] employed EOG modalities to detect drowsiness; in [200] several emotions-happiness, sadness, anger, fear, and satisfaction-were detected purely based on the eye motion. AttentivU [118] is a prominent example of multimodal affective computing. The combination of EOG and EEG electrodes in a socially acceptable pair of glasses creates an opportunity for seamless mental state monitoring: attention tracking for educational purposes [81], meditation [178], or driving [190]. More importantly, the inconspicuous integration of ExG electrodes into the traditional glasses form-factor paves the way for the integration of affective computing with XR applications [64].

Biometrics and Security in XR. Existing authentication techniques in XR rely on input methods with low throughput (e.g., mid-air virtual keyboards) and are vulnerable toward observer attacks (e.g., virtual objects selection), as noted in References [89, 136, 175]. EEG-based biometrics can offer an alternative method for establishing trust in XR systems, enabling functionalities such as user authentication, password protection, and secure online purchases. EEG captured even by consumer-grade devices offers a secure tool for biometric authentication [126]. EEG-based authentication procedures can function seamlessly without requiring the user's attention (e.g., resting with eyes open) and are inherently less vulnerable to external observation. Moreover, they are universal and permanent for individuals, hard-to-replicate and potentially cancellable [137] (i.e., can be replaced once compromised), and are built upon EEG signals that cannot be easily extracted without the user's knowledge [91]. However, using EEG-based authentication in XR headsets has its own limitations. In general, in XR users move, gesticulate, and interact with controllers. This induces electrical and mechanical noise that inevitably corrupts EEG signals and creates artifacts. Due to this, the users' activity during the authentication process must be significantly restrained: users have to follow strictly defined procedures for a certain amount of time [91]. Existing works $[91,136]$ rely on predefined authentication procedures, e.g., a series of images (such as animals or celebrities) are presented to the user; consequently the evoked potentials are used for authentication. Combined with the unavoidable discomfort of wearing a HMD with EEG electrodes, this might negatively affect user experience and hinder widespread adoption. However, unobtrusive electrodes [64] and personalizable routines lessen the burdens of EEG-based biometrics.

sEMG signals are reliable biometric modalities too. Fan et al. [83] proposed a way to unlock smartphones based on patterns of sEMG arising in users' forearm muscles while they pick up smartphones using a Siamese neural network that extracts distinctive key patterns from pairs of sEMG envelopes from different users. Usage of the myoelectric signature of a natural gesture to devise an authentication system can be applied to interaction with XR (e.g., sEMG of lifting an arm to access a touch surface of AR glasses). In that case, sEMG authentication is more subtle and does not require to perform any specific actions or mental efforts (compared to EEG-based).

An alternative approach to authentication in XR head-mounted displays relies on unique features of the human visual system, such as eye globe, eyelid, and extraocular muscle movements. OcuLock [142], a system designed specifically for HMDs, captures the EOG associated with the ocular activity. Visual stimuli, presented in an immersive VR environment, improve the robustness of authentication using the acquired signal. OcuLock, as an EOG-based authentication system, is resistant to impersonation and statistical attacks. As a part of the system's evaluation, Luo et al. [142] conducted a questionnaire that established: (i) users prefer dynamic and attractive visual stimuli in VR, and (ii) EOG-based authentication system is a more convenient and socially acceptable method of establishing trust in XR, compared to gesture- and brainwave-based approaches.

When the blurred interfaces have inclined to our reality [106], such tools for monitoring our levels of comprehension to unfamiliar texts could be extended to the physical counterparts, and even makes the human user more awareness to the environments [214]) with better cognitive 


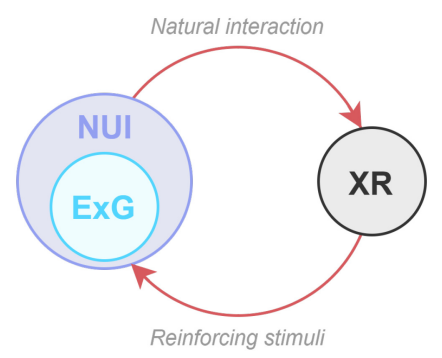

Fig. 16. Two-way interaction ExG-XR.

powers [66], as many existing works propose augmented information on texts [50], daily objects [199], and office environments [97].

\section{TWO-WAY INTERACTION: CLOSING THE LOOP}

In the previous section, the ways how ExG-based interfaces can enrich interaction in XR have been discussed. However, the tackled concept of two-way interaction implies a bidirectional relationship (see Figure 16): ExG-based interfaces advance natural interactions in XR and, simultaneously, immersive realities positively reinforce the interaction itself. Extended realities render a realm, where visual stimuli enhance user experience and elicited responses [70]; a realm that allows more granular control over environs and parameters of the experience [117, 119].

As shown by Balban et al. [70], virtual reality activates the genuine threat detection/response mechanism in humans. In particular, realistic representation of heights increased the arousal and visual scanning. Compared to traditional visual stimuli in neurophysiological research (still images and videos), immersive stereoscopic imagery triggered natural behavioral responses, that were captured by an invasive BCI. The finding is clearly feasible over the entire reality-virtuality continuum. Additionally, as noted in Reference [142] XR generally improves the robustness of biosignal classification, while vivid and immersive imagery is preferred by users. XR provides a great opportunity for the development of down-to-earth scenarios with practical scenes that supports complex user interaction in daily life. However, closing the loop between XR and ExG-driven NUIs needs a high degree of completeness in terms of naturalistic and ergonomic interactions. Immersive realities transform into an environment of deeper emotional connection between humans, offering a platform for engaging communication, activating a stronger emotional reaction. It is especially important in the age of remote collaborations and distance interconnections [63].

Extended realities offer adjustable yet stable and continuous environments. In cognitive and social sciences, VR experiences are applied to address the issue of ecological validity, when the functional and factual similarity between clinical and real-life results does not hold [119]. Controlling the variables of virtual experiments, researchers are empowered to deliver reproducible and reliable results. In the context of ExG-driven interfaces, the underlying dynamics of neural circuitry is more stable and predictable when user movements are restricted by a virtual scene.

It is also worthwhile to mention that XR environments are a technological enabler in which the ExG practitioners can tamper parameters of the experiments. The Protheus effect [117], which can be described as conformity toward digital avatars, shows how immersive realities can substitute certain aspects of reality while inducing a genuine response. By tampering physical properties of a user's avatar in XR (e.g., height, skin color, limb configuration) a change in the user's mentality can be achieved, such as the elimination of racial bias [57]. We foresee that the further integration of immersive and ExG-driven NUIs can be accomplished by a visionary symbiotic combination of XR and bio-electricity sensing technologies [20] (see also Figure 17). 


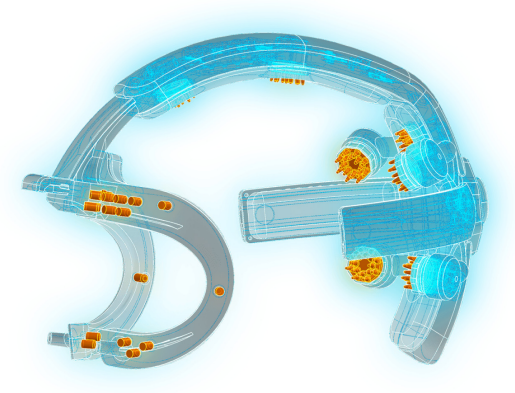

Fig. 17. Project Galea [21].

Table 3. Familiarity of Questionnaire Participants with AR, VR, and Novel $\mathrm{HCl}$

\begin{tabular}{lrrr}
\hline & AR & VR & Novel HCI \\
\hline (1) It is the first time I hear about this. & $4.3 \%$ & $0 \%$ & $40.9 \%$ \\
(2) I have heard about/seen that. & $51.6 \%$ & $35.5 \%$ & $29 \%$ \\
(3) I have some experience with that. & $30.1 \%$ & $50.5 \%$ & $17.2 \%$ \\
(4) I do use this technology on regular basis. & $4.3 \%$ & $7.5 \%$ & $4.3 \%$ \\
(5) I do research in that field / I develop solutions in this area. & $9.7 \%$ & $6.5 \%$ & $8.6 \%$ \\
\hline
\end{tabular}

\section{A QUESTIONNAIRE ON SOCIAL ACCEPTANCE OF NOVEL USER INTERFACES}

To probe the factor of social acceptance of the ExG-based novel NUIs, we design, evaluate and present an anonymous online questionnaire. We adopt the methodology discussed by Schwind et al. [193], that also establishes the feasibility of such a survey in the context of social acceptance of novel technology. As formulated by DeWall et al. [78], social acceptance toward a technology can be defined as a desire to include a person using it into social interactions; while rejection can be defined as an communicative exclusion of such individuals.

As argued by Montero et al. [158], while conducting an inquiry into acceptance of gestural only interfaces, there should be distinction between two scenarios: when (1) participants are willing to use a particular NUI themselves or (2) are willing to interact with bystanders who are utilizing them. Besides role-based acceptance, Montero et al. add a privacy-related dimension by differentiating between utilizing gesture-based interfaces at home (private context) or in public. Their study estimates social acceptance of various interaction gestures in private and public contexts by asking respondents to assign a soft label from 1 (embarrassed) to 6 (comfortable) to a gesture in a context. The results of the survey show that suspenseful gestures (such as writing big letters in the air) were not perceived as acceptable, while "magic" gestures (such as controlling a presentation or a smart home device) and secretive ones (subtle tapping) were accepted by target study groups.

In our questionnaire, we introduce ExG-based NUI modalities one by one. For each modality (BCI, Muscle-based inputs, SSI, and EOG), we provide a short description, examples of usage scenarios and visual illustrations [177]. Initially, we asked the participants to indicate their experience with augmented reality, virtual reality and novel HCI interfaces by selecting one of five possible answers with (1) to indicate that it is the first time they hear about this and (5) to indicate that they do research in that field, as presented in Table 3.

Furthermore, $92 \%, 91 \%$, and $90 \%$ of the participants who either do research in or use VR, AR, or novel $\mathrm{HCI}$ interfaces daily respectively, state that they are willing to adopt $\mathrm{BCI}$ as an alternative 


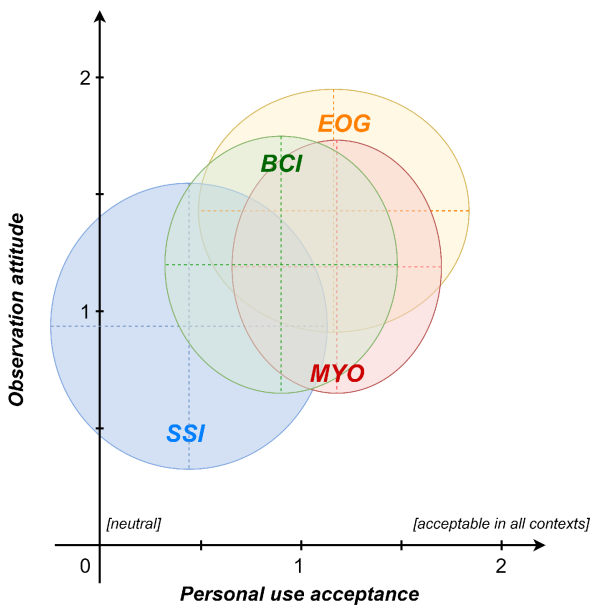

Fig. 18. Graphical representation of questionnaire result: Each ellipse depicts a range ( -2 to 2$)$ of acceptance labels toward a certain NUI modality in coordinates of personal usage and observing acceptance.

input method either at home or in public and are indifferent if anyone is using such inputs around them. However, the responses of participants who stated that they are not familiar with novel HCI interfaces vary when asked about their willingness to accept users in their home and they will adopt such technologies only once they are mainstream. Additionally, $39 \%$ of the participants stated that they are comfortable with using BCI interfaces, silent speech interfaces and gaze control interfaces either at home or in public.

We then ask two main questions on the acceptance of the technology in terms of ownership (personal usage) and observing scenario (bystander). We differentiate between the willingness of using the technology in the private and public contexts, assigning labels in the range of $[-2 ; 2]$, where (-2) stands for rejection in every context, (-1) in one of them, (0) for neutral, (1) and (2) for acceptance in one or every context, respectively. The questionnaire was filled by 93 participants, who were asked to indicate their prior knowledge on XR technologies and NUIs.

As depicted in Figure 18, we represent each modality as an ellipse in $[x, y]$ coordinates, where the $x$ axis shows the label of personal usage acceptance and the $y$ axis observing acceptance of modality. Center of each ellipse shows the average acceptance label, while standard deviation of respondent's acceptance determines the size of it. It clear that given such representation all modalities are located in the "acceptance" coordinate quarter with silent speech interfaces being the least and eye tracking the most preferred modalities.

It is important to note that the majority of the participants who gave a positive rating to ExG wearables are not willing to purchase them before they become mainstream. This implies that the consumers are more willing to employ ExG wearable after the first mover appears in the commercial market. We conjecture that such ExG wearable can be quickly accepted by the consumers once the technology giants (e.g., Apple, Samsung, Huawei) create their pilot user groups and cultivate consumer awareness to such ExG-supported interfaces for the immersive technology. However, XR headsets have been primarily employed at workplaces and nowadays show very limited usage in the mass market. Good incentives exist for XR headsets to seek an alternative way for productivity in a new form of work spatiality and the EXG devices work complementary with the XR headsets by supporting hands-free interaction. For example, laborers in warehouses get rid of handheld devices with the mobile handsets and ExG two-way user interaction channels and thus reserve two free hands for fast item picking in their routine operations. The questionnaire results also 
demonstrate that the respondents would not buy the ExG wearable, however, they would like to use it if the devices are available. Thus, we see that the likelihood of adopting ExG with XR headsets at the workplace remains high if their employers offer the devices in the working environments.

\section{CHALLENGES AND OPEN PROBLEMS}

ExG input modalities are facing several challenges. For example, BCI suffers from the low strength of the acquired biological signals, as they are recorded indirectly; localization of the source of the certain cortical activity is a challenge in EEG due to the smearing of the signal. High-quality signal amplification can address this problem, improving the precision of the analysis. The wide adoption of invasive interfaces is very unlikely, but there exist commercial-driven research in the direction of embedding wires under the skin surgically for precise high-speed BCIs [163]. However, user safety and social acceptance are the key concerns in that case. Additionally, the performance of such BCI will deteriorate over time as biological tissues will inevitably die off.

The shifting and non-constant nature of biological signals, recording errors, interference, and inaccurate signal classification contribute to overall high error rates. Given that, ITRs of most BCI systems are very low compared to classical I/O methods, thus minimizing errors in the acquisition and pre-processing stages becomes crucial. Furthermore, the Midas touch problem affects most of the ExG-based NUI modalities: when an unwanted input by the user that performs a natural nonHCI-related activity is recognized as valid input. In eye-tracking systems, the user might move his eyes away from the computer screen, yet the tracking system will recognize some of the eye gestures for input. The deliberate interaction of eye movements should be distinguishable by the computer system. Similarly, intrinsic processes of thought and motor control will affect the performance of mobile BCI in unconstrained scenarios. Several solutions have been proposed for gaze tracking $[68,207]$, since this problem can be addressed by HCI applications and protocols.

In addition to the technical problems of NUI, several ethical concerns regarding NUI were raised as well [111]. EEG, for example, records signals of the whole brain including cognitive activities, i.e., thinking. Thus some people might not approve of their EEG data being accessed by anybody else. Similarly, sEMG data can be used to identify subjects, inflating the privacy issues of ExG. Nevertheless, there are areas where ExG NUI can hardly be replaced. Rehabilitation methods, prosthetic devices, and assistive technologies heavily rely on EEG and EMG recordings especially in the case of locked-in patients. Consecutively, NUIs are the only option for the disabled to interact with computing systems, including XR.

\subsection{Future Directions}

As illustrated in Section 4, the area of application of ExG-based is expanding beyond rehabilitation, prosthesis, and adjacent research, offering exciting opportunities for end-users to interact with interfaces daily. In particular, the combination of ExG and XR makes virtual worlds more immersive and adaptive by enriching two-way interactions and simultaneously alleviating the need for physical controllers [25, 198]. On the roadmap toward wider adoption of the discussed ExG-based input paradigms into intelligent user interfaces for the general users, we envision three major milestones, including hardware (mobile design, sensor adoption, and integration), software (signal filtering, processing, and classification), and user experiences (transforming interfaces, interaction, and experiences).

Sensors toward High Mobility. Current issues and challenges of ExG hardware (as discussed in Section 3.5) can be addressed by creating sensors that are smaller, more universal, more accurate, and more resistant to noise. Thus, the development and adoption of ExG-enabled solutions might be pushed further, which aligns with the purpose of ubiquitous interaction through XR mobile 
headsets. Moreover, the progress of mobile batteries can make it possible for sensors to work autonomously for extended periods of time, this can provide a substantial increase in mobility for patients that are tied to their medical equipment. In addition, progress in the technologies of wireless communications can lead to the manufacturing of less energy demanding sensors that are more flexible and require less attention from users to reconfigure connections and seamlessly add new sensors [64].

Multi-sensory Signal Aggregation. As the sensors become more mobile and noise resistant, the acceptance by the XR end-users is increased, followed by the supply of integration of XR with ExG electrodes [14, 25, 64]. From a biological perspective EEG, EMG, and EOG signals are different, but can there be a universal software platform to effectively analyze these signals? That will help to smoothly integrate different modalities into more effective hybrid interfaces. If, for example, electrodes location can be detected by properties of the acquired signal, then users will be spared from the burden of tedious configuration. Will the ongoing process of hardware miniaturization and further advances in machine learning techniques lead to the creation of more accurate and more precise sensors? Such developments would promote the acceptance and usage of ExG-enabled solutions for two-way user interaction between users and XR headsets.

ExG on Electronic Textile. The research community proposes Electronic textile (E-textile) primarily for smart garments. Among the most recent works, the embedded sensors in E-textiles such as strain [82] or kinetic [93] sensing and conductive stretchable fabrics [140] support the interaction between human users and mobile computers through parsing the body gestures into stitch geometries or joint movement. E-textile can also serve as a large-area touch sensible areas on smart garments [212], to sustain the decade-old touchscreen interaction on AR/VR head-worn computers. More importantly, ExG sensors can be designed as flexible textile electrodes [43] embedded in the spacious smart garment. Several projects already presented incorporated ExG electrodes: for tracking muscle performance [3] and rehabilitation [176]. Such an embedding makes the acquisition of ExG signals less obtrusive shifting the social acceptance of the ExG-based interfaces.

Deep Learning Approach to Bio-signal Processing. The area of bio-signal processing is wast and historically mature. The history of BCI, for example, is almost 50 years, with first studies conducted in the early 1970s [182]. A review of state-of-the-art algorithms used in bio-signal processing, such as Fourier Analysis, Power Spectrum Density feature extraction alongside many others, can be found in Reference [47]. The classical approach to signal processing is not demanding computationally but requires precise feature engineering and manual threshold adjustments. In recent years, machine learning has revolutionized multiple areas including computer vision, natural language processing, and ubiquitous computing. Advanced deep neural networks found their ways into self-driving cars, autonomous flying drones, a variety of IoT devices, and many more. The deep learning approach has been adopted recently in the area of $\mathrm{BCI}$ and other bio-signal processing as well; an overview of deep learning techniques applied for healthcare and physiological signal processing is presented in Reference [86]. Works, discussed in this survey, that employ deep learning classifiers are presented in Table 4. In general, the advent of deep learning, improved the transferability of algorithms, relieving researchers and practitioners in the area of bio-signal analyses from the burden of feature-handcrafting, making the ExG modalities a feasible input for hands-free interaction with XR headsets [131].

Redefining Interfaces. Existing NUIs with low ITRs can be used to provide an input of several control commands in mobile HCI scenarios: 2 labels in accept/decline call; replying instant messages or emails with on of the several predefined templates; item selection in menus; feed scrolling with navigation UI elements (e.g., SSVEPs markers in the edges of the screen). In recent years, there is a 
Table 4. Deep Learning Classifiers for Bio-signal Processing

\begin{tabular}{llllc}
\hline Study & Modality & Application & \multicolumn{1}{c}{ Applied classifier } & Accuracy \\
\hline$[111]$ & SSI & HCI & 1D CNN: 3 CL, 1 FCL, softmax & $92 \%$ \\
{$[224]$} & EMG & Prosthesis & 2D CNN : 1 CL, 2 FCL, softmax & $65 \%$ \\
{$[54]$} & EMG & Prosthesis & 2D CNN: 5 CL, 1 FCL, softmax & $66 \%$ \\
{$[210]$} & SSI & HCI & DNN: 4 FCL + HMM & N/A \\
{$[165]$} & BCI, SSVEPs & Speller & 1D CNN: 4 CL, 1 FCL, softmax & $99 \%$ \\
{$[229]$} & BCI, MI & Robot control & LSTM classifier & $93 \%$ \\
{$[59]$} & EMG & HCI & CNN: 2 CL, 1 FCL, 3 LSTM, softmax & $97 \%$ \\
{$[228]$} & BCI, MI & HCI, Speller & CNN: 2 CL, 2 FCL, softwmax + RNN: 4 & $95.53 \%$ \\
& & & FCL, 2 LSTM cells, softmax + 3 FCL & \\
{$[231]$} & EOG & HCI, Drowsiness & CNN: 2 CL + linear regression & N/A \\
\hline
\end{tabular}

CNN, Convolutional Neural Network; CL, convolutional layer; FCL, Fully Connected layer; RNN, Recurrent Neural Network.

trend toward lean and simplified graphical interfaces on the mobile headsets and wearables [125]. Limited bandwidth of input devices becomes a constraint to the design of a GUI. Complicated interfaces with multiple densely placed objects are not favorable for mobile scenarios. Thus, a lot of research opportunities exist for designing ExG-based NUI-driven user interfaces. Simultaneously, faster NUIs, like EMG, see Figure 19, can be used as alternative text input methods, for swift navigation within GUI or virtual spaces. Advancements in SSI, once this technology becomes more convenient, accurate, and accepted by the community, may lead to the formation of synthetic telepathy networks, where intended speeches (even within limited vocabulary) can be effectively obtained and securely transmitted to a recipient or broadcast to a group of audiences [156]. While the research prototypes for two-way interactions with XR headsets primarily rely on touch-based and vision-based approaches (Section 1.2), the emerging input modals of ExG have not been fully explored. Importantly, the user interaction in XR goes beyond the WIMP desktop metaphor. And huge efforts should be made to explore the seamless user interaction with ExG in XR that includes both the WIMP-based and reality-based interfaces. It is necessary to narrow down the ITR gaps between ExG-based NUIs and traditional interfaces, to fulfill the aforementioned user interaction in the enriched XR.

Optimizing Interaction. According to Reference [146], mobile users on average spend 3 hours and 15 minutes per day on their smartphones, while the top $20 \%$ of smartphone users have been recorded the daily screen time more than 4.5 hours. Among this time, 2 hours and 22 minutes on average are spent on socializing online through the six most popular platforms including Facebook, Twitter, and Instagram [46]. These social media apps on smartphones have organized the contents on the reverse-chronological timelines and the swipe gesture enables the smartphone users to navigate the contents and to scroll over news feeds horizontally [202]. Similarly, the size-constrained Google Glass [100], which is the first-ever commercial AR head-worn computer debuted in 2013, can only accommodate a tiny touchpad on the spectacle frame of the smart glasses, and hence the operating system displays the information using Timeline, in which the users swipe over the pixel cards horizontally and select the targets. Thus, one of the future directions is to investigate the timeline-based interaction supported by low-ITR NUIs, and BCIs in particular. While the traditional interface design of the BCI systems mainly relies on the point-and-click interaction paradigm for target acquisition [169], novel interfaces can employ scroll-driven timelines. It is important to note that the scroll-driven timeline has limited capabilities to handle very 


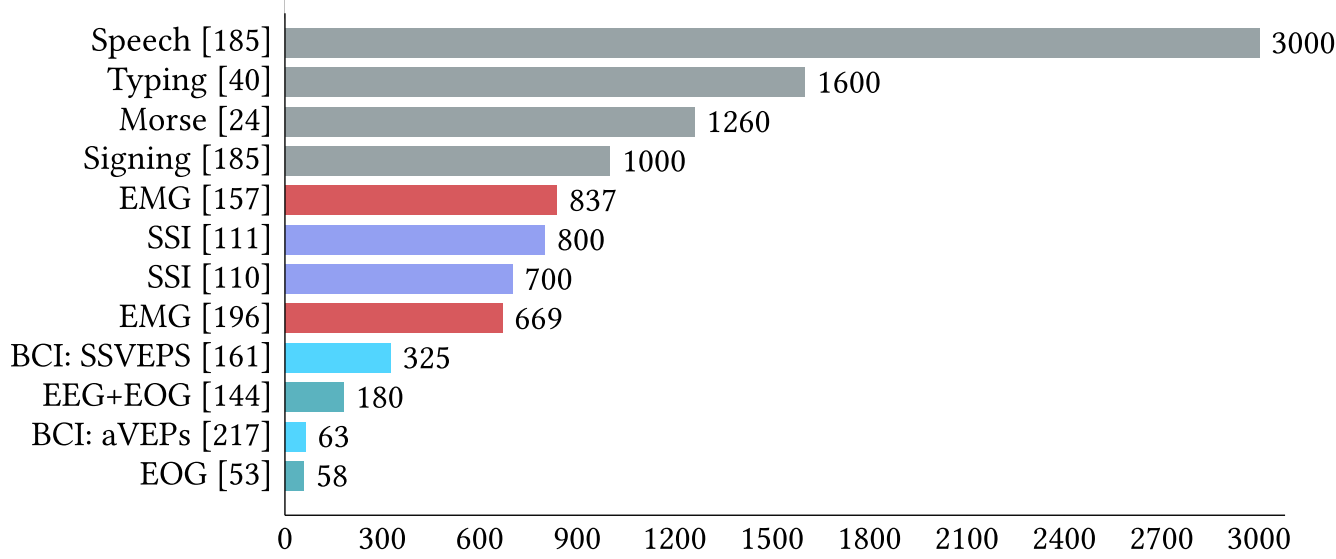

Fig. 19. ITR, in bits per minute, of communication modalities; Showing decreasing ITR from traditional interfaces (Speech and Typing) to the NUIs.

complicated and voluminous information. The display of such information can be further optimized by the context-aware architectures [125].

Fashionable ExG. Trends show that ExG sensors can be embedded in smart wearables, and several commercial products already exist employing ECG sensor mesh on the sports T-shirts [164]. Smart bands, watches, other wearable devices, and even jewelry may be equipped with ExG sensors in the future. To enhance the user acceptability, ExG electrodes can be seamlessly introduced into sports [166] or driving helmets and glasses [104], providing distraction-free inputs and state-monitoring (e.g., drowsiness), or incorporated into winter headdress for places with cold climate when touch-enabled input is ineffective due to low temperatures and voice interfaces are obstructed by garments [110].

\section{CONCLUSION}

As stated in the book Brave NUI World, the landscape of designing natural interfaces pushing the bandwidth to the limit have never stopped changing, since the popularity of computing devices and the advancement of sensor technology. Computing devices of today have been shrunken to wearable size (e.g., wrist-worn and head-worn) and are now facing the challenges of interaction through diminished user interfaces. Thus, we stand at the brink of another potential shift toward more concealed modalities and body-centric interfaces enabled by leveraging the latest ExG devices.

In this survey, we presented an overview of emerging ExG modalities. Starting from the biological foundations, we have explained the genesis of biological signals: neural activity in the brain, retino-corneal potentials in the eye, myoelectric currents in muscles, and speech production. Following, bio-signal acquisition techniques are mentioned as well as hardware for such an acquisition. We outlined the potential of mobile usage of imaging devices and aspects of combining these devices with XR equipment. Next, we demonstrated how ExG modalities are already applied and can be applied for interactions with extended realities. We manifested five major directions: subtle interactions, interactions with the real-world objects and virtual objects, affective computing, and biometrics-where ExG-based NUIs can enrich two-way interaction between immersive realities and end-users. We have introduced the concept of two-way interaction between ExG interfaces and extended realities: ExG-based interfaces augment interaction with immersive realities, while 
$\mathrm{XR}$ reinforces the interaction by providing controllable environments and more intense stimuli. To back the trend of integration of XR and ExG modalities, we conducted a questionnaire on social acceptance of novel interaction paradigms and presented its results. The results showed that the majority of respondents shown a positive attitude toward muscle-powered, silent speech, braincomputer, and gaze-based interfaces. We concluded the article with a sketch of a roadmap toward more intuitive, user-friendly, and effective ExG-based interfaces for XR and mobile computing. Reflecting back to the discussed metric of ITR, we've shown a comparative summary of ITRs of the discussed NUIs and other communication modalities, such as typing tasks (Figure 19). To conclude, we believe such an overview will be helpful to those who are interested to start applied research in the area of NUI at the frontier of extended realities. 
APPENDICES

A PORTABLE EEG HEADSETS

Table 5. Portable EEG Headsets. Part 1

\begin{tabular}{|c|c|c|c|c|}
\hline Electrodes: & $\begin{array}{l}\text { 1. EMOTIV } \\
\text { EPOC+ [16] }\end{array}$ & $\begin{array}{l}\text { 2. EMOTIV } \\
\text { Insight } 5 \text { [17] }\end{array}$ & $\begin{array}{l}\text { 3. AEPOC Flex } \\
\text { Saline Sensor } \\
\text { Kit [19] }\end{array}$ & $\begin{array}{l}\text { 4. EPOC Flex Gel } \\
\text { Sensor Kit [18] }\end{array}$ \\
\hline Channels: & $\begin{array}{c}\text { AF3, F7, F3, FC5, } \\
\text { T7, P7, O1, O2, P8, } \\
\text { T8, FC6, F4, F8, } \\
\text { AF4 }\end{array}$ & $\begin{array}{c}\text { AF3, AF4, T7, T8, } \\
\text { Pz }\end{array}$ & Configurable & Configurable \\
\hline $\begin{array}{r}\text { Additional } \\
\text { Sensors: }\end{array}$ & $\begin{array}{l}\text { Magnetometer, } \\
\text { Accelerometer }\end{array}$ & $\begin{array}{l}\text { Gyroscope, } \\
\text { Magnetometer, } \\
\text { Accelerometer }\end{array}$ & $\begin{array}{l}\text { Magnetometer, } \\
\text { Accelerometer }\end{array}$ & $\begin{array}{l}\text { Magnetometer, } \\
\text { Accelerometer }\end{array}$ \\
\hline Resolution: & $\begin{array}{c}14 \text { or } 16 \text { and } 16 \\
\text { bits }\end{array}$ & 14 bits and 14 bits & 14 bits & 14 bits \\
\hline $\begin{array}{r}\text { Sampling } \\
\text { rate: }\end{array}$ & $2048 \rightarrow 256$ or 128 & 128 & $1024 \rightarrow 128$ & $1024 \rightarrow 128$ \\
\hline Protocol: & BLE & BLE & $\begin{array}{c}\text { Proprietary } \\
\text { wireless protocol } \\
\text { @ } 2.4 \mathrm{Ghz} \\
\end{array}$ & $\begin{array}{c}\text { Proprietary } \\
\text { wireless protocol } \\
\text { @ } 2.4 \mathrm{Ghz} \\
\end{array}$ \\
\hline Battery: & $\begin{array}{c}\text { 640mAh: } 12 \mathrm{~h} \\
\text { (USB receiver), } 6 \mathrm{~h} \\
\text { BLE }\end{array}$ & $\begin{array}{l}\text { 480mAh: } 8 \mathrm{~h} \text { (USB } \\
\text { receiver), } 4 \mathrm{~h} \mathrm{BLE}\end{array}$ & 640mAh: $9 \mathrm{~h}$ & 640mAh: $9 \mathrm{~h}$ \\
\hline Device: & 5. Neurosky [37] & $\begin{array}{c}\text { 6. Muse } \\
(2014)[31]\end{array}$ & $\begin{array}{l}\text { 7. Muse } 2(2016) \\
{[30]}\end{array}$ & $\begin{array}{c}\text { 8. mBrainTrain } \\
\text { SMARTING } \\
24[38]\end{array}$ \\
\hline Electrodes: & 1 dry & 4 dry & 4 dry & 24 dry \\
\hline Channels: & FP1 & $\begin{array}{l}\text { TP9, AF7, AF8, } \\
\text { TP10 }\end{array}$ & $\begin{array}{c}\text { TP9, AF7, AF8, } \\
\text { TP10 }\end{array}$ & $\begin{array}{l}\text { FP1, FP2, AFz, F7, } \\
\text { F3, Fz, F4, F8, T7, } \\
\text { C3, Cz, C4, T8, } \\
\text { M1, CPz, M2, P7, } \\
\text { P3, Pz, P4, P8, } \\
\text { POz, O1, O2 }\end{array}$ \\
\hline $\begin{array}{r}\text { Additional } \\
\text { Sensors: } \\
\end{array}$ & - & Accelerometer & $\begin{array}{c}\text { PPG, } \\
\text { Accelerometer }\end{array}$ & Gyroscope \\
\hline Resolution: & 12 bits & 10 or 16 bits & 10 or 16 bits & 24 bits \\
\hline $\begin{array}{r}\text { Sampling } \\
\text { rate: }\end{array}$ & 512 & $220 \mathrm{~Hz}$ or $500 \mathrm{~Hz}$ & $256 \mathrm{~Hz}$ & $250 \mathrm{~Hz}$ or $500 \mathrm{~Hz}$ \\
\hline Protocol: & BT/BLE & BT 2.1 + EDR & BT 4.0 and BLE & BT $2.1+$ EDR \\
\hline Battery: & $\begin{array}{l}\text { 1000mAh (AAA } \\
\text { Battery): 8-hours }\end{array}$ & 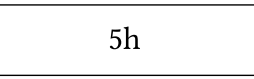 & 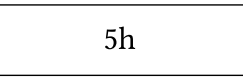 & $560 \mathrm{mAh}: 4 \mathrm{~h}$ \\
\hline
\end{tabular}


Table 6. Portable EEG Headsets. Part 2

\begin{tabular}{|c|c|c|c|c|}
\hline Device: & 9. cEEGrid [8] & $\begin{array}{l}\text { 10. Cognionics } \\
\text { QUICK }\end{array}$ & $\begin{array}{l}\text { 11. Cognionics } \\
\text { Mobile }\end{array}$ & $\begin{array}{l}\text { 12. Auris In-Ear } \\
\text { EEG [4] }\end{array}$ \\
\hline Electrodes: & 18 dry & $8 / 20 / 30$ dry & $64 / 128$ wet & $\begin{array}{c}2 \text { dry (+6 via } \\
\text { extension) }\end{array}$ \\
\hline Channels: & $\begin{array}{l}\text { Custom locations } \\
\text { around ears }\end{array}$ & $\begin{array}{l}\text { Configurable (hot } \\
\text { placement) }\end{array}$ & Configurable & $\begin{array}{l}\text { Left, Right ear and } \\
\text { Chest ECG }\end{array}$ \\
\hline $\begin{array}{r}\text { Additional } \\
\text { Sensors: }\end{array}$ & Gyroscope & p & $\begin{array}{l}\text { Accelerometer, } \\
\text { Gyroscope }\end{array}$ & - \\
\hline Resolution: & 24 bits & 24 bits & 24 bits & 24 bits \\
\hline $\begin{array}{r}\text { Sampling } \\
\text { rate: }\end{array}$ & 250 or $500 \mathrm{~Hz}$ & $\begin{array}{c}500 \mathrm{~Hz} \text { or } 1000 \mathrm{~Hz} \\
\text { or } 2000 \mathrm{~Hz}\end{array}$ & $500 \mathrm{~Hz}$ or $1000 \mathrm{~Hz}$ & $\begin{array}{c}250 \text { or } 500 \text { or } \\
1,000 \text { or } 2,000 \mathrm{~Hz}\end{array}$ \\
\hline Protocol: & BT 2.1 & $\mathrm{BT}$ & BT & BT \\
\hline Battery: & & $\begin{array}{l}\text { 8h (wireless); 16h } \\
\text { (microSD card) }\end{array}$ & $\begin{array}{l}\text { 6h (wireless); 8h } \\
\text { (microSD card) }\end{array}$ & $\begin{array}{l}\text { 8h (wireless); } 16 \mathrm{~h} \\
\text { (microSD card) }\end{array}$ \\
\hline Device: & $\begin{array}{l}\text { 13. Cognionics } \\
\text { DevKit [9] }\end{array}$ & $\begin{array}{c}\text { 14. Open BCI } \\
\text { Ultracortex "Mark } \\
\text { IV" EEG Headset + } \\
\text { Cyton Biosensing } \\
\text { Board [32] }\end{array}$ & $\begin{array}{c}\mathbf{1 5 .} \\
\text { DSI-VR300 [14] }\end{array}$ & $\begin{array}{l}\text { 16. LooxidVR } \\
\text { Headset \& } \\
\text { Mask [25] }\end{array}$ \\
\hline Electrodes: & 8 dry & $\begin{array}{c}8 \text { dry (+8 via } \\
\text { extension) }\end{array}$ & 7 dry & 6 dry \\
\hline Channels: & $\begin{array}{l}\text { Configurable } \\
\text { around the } \\
\text { headband }\end{array}$ & $\begin{array}{c}\text { Configurable, } \\
\text { chassis can be } 3 \mathrm{D} \\
\text { printed }\end{array}$ & $\begin{array}{c}\mathrm{Fz}, \mathrm{Pz}, \mathrm{P} 3, \mathrm{P} 4, \mathrm{PO} 7 \\
\mathrm{PO} 8, \mathrm{Oz}\end{array}$ & $\begin{array}{l}6 \text { electrodes over } \\
\text { the frontal lobe, } \\
\text { custom positions }\end{array}$ \\
\hline $\begin{array}{r}\text { Additional } \\
\text { Sensors: }\end{array}$ & Configurable & Accelerometer & $\begin{array}{c}\text { Optional } \\
\text { embedded 3D } \\
\text { accelerometers }\end{array}$ & $\begin{array}{l}\text { Eye tracking } \\
\text { camera (eye } \\
\text { movement, and } \\
\text { pupil dilation), } \\
\text { IMU }\end{array}$ \\
\hline Resolution: & 24 bits & 24 bits & 16 bits & 24 bits \\
\hline $\begin{array}{r}\text { Sampling } \\
\text { rate: }\end{array}$ & $\begin{array}{c}250 \text { or } 500 \text { or } \\
1,000 \text { or } 2,000 \mathrm{~Hz}\end{array}$ & $250 \mathrm{HZ}$ & $\begin{array}{c}300 \mathrm{~Hz}(600 \mathrm{~Hz} \\
\text { option) }\end{array}$ & $1000 \mathrm{~Hz}$ \\
\hline Protocol: & BT & BLE & BT & BT \\
\hline Battery: & $\begin{array}{l}\text { 8h (wireless); } 16 \mathrm{~h} \\
\text { (microSD card) }\end{array}$ & $\begin{array}{c}4000 \mathrm{mAh} \text { (4 AAA } \\
\text { batteries) }\end{array}$ & $12+\mathrm{h}$ & $\begin{array}{l}\text { Requires external } \\
\text { source (USB }+5 \mathrm{~V} \\
\text { DC) }\end{array}$ \\
\hline
\end{tabular}


Table 7. Portable EEG Headsets. Part 3

\begin{tabular}{|c|c|c|c|c|}
\hline Electrodes: & $\begin{array}{c}\text { 17. B-Alert } \\
\text { X24/X10 EEG } \\
\text { System [5] }\end{array}$ & 18. BR32S [6] & 8 & $\begin{array}{l}\text { 20. Mindo } \\
\text { Jellyfish/Trilobite/ } \\
\text { Coral [26] }\end{array}$ \\
\hline Channels: & $\begin{array}{c}\text { Fz, F3, F4, Cz, C3, } \\
\text { C4, POz, P3, P4 / } \\
\text { Fz, F1, F2, F3, F4, } \\
\text { Cz, C1, C2, C3, C4, } \\
\text { CPz, Pz, P1, P2, P3, } \\
\text { P4, POz, Oz, O1, } \\
\text { O2 }\end{array}$ & $3210-20$ system & $\begin{array}{c}\mathrm{Fp} 1, \mathrm{Fp} 2, \mathrm{Fz}, \mathrm{C} 3 \\
\mathrm{C} 4, \mathrm{Pz}, \mathrm{O} 1, \mathrm{O} 2\end{array}$ & $\begin{array}{c}\text { AF7, Fp1, Fp2, } \\
\text { AF8 / } 32 \text { 10-20 } \\
\text { system / } 64 \text { 10-20 } \\
\text { system }\end{array}$ \\
\hline $\begin{array}{l}\text { Additional } \\
\text { Sensors: }\end{array}$ & $\begin{array}{c}\text { 3-axis } \\
\text { accelerometer }\end{array}$ & - & - & - \\
\hline Resolution: & 16 bits and 12 bits & 16 bits & 16 bits & 24 bits \\
\hline $\begin{array}{r}\text { Sampling } \\
\text { rate: }\end{array}$ & $256 \mathrm{~Hz}$ & $250 \mathrm{~Hz}$ & $250 \mathrm{~Hz}$ & $500 \mathrm{~Hz}$ \\
\hline Protocol: & BT & BT 2.1 & BT 2.1 & BT \\
\hline Battery: & $8+h$ & $10+\mathrm{h}$ & $10+h$ & - \\
\hline
\end{tabular}

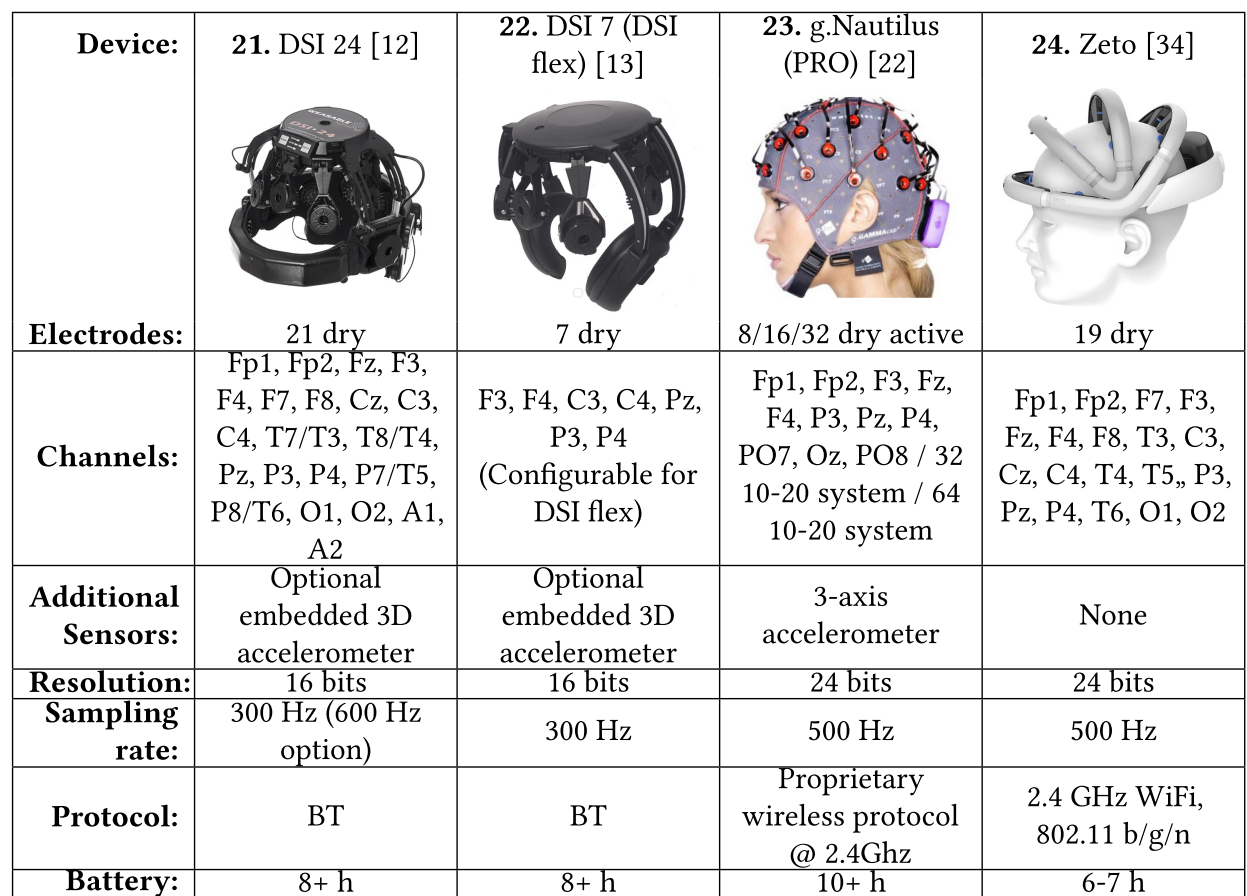


Table 8. Portable EEG Headsets. Part 4

\begin{tabular}{|c|c|c|c|c|}
\hline Device: & 25. Hero [23] & 26. Diadem [11] & 27. Air [2] & 28. NextMind [10] \\
\hline Electrodes: & 9 dry & 12 dry & 8 dry & 9 dry \\
\hline Channels: & $\begin{array}{c}\mathrm{FC} 3, \mathrm{FC}, \mathrm{FC} 4, \mathrm{C} 3 \\
\mathrm{Cz}, \mathrm{C} 4, \mathrm{CP} 3, \mathrm{CPz} \\
\mathrm{CP} 4\end{array}$ & $\begin{array}{c}\text { Fp1, Fp2, AF7, } \\
\text { AF8, F3, F4, P3, P4, } \\
\text { PO7, PO8, O1, O2 }\end{array}$ & $\begin{array}{l}\text { Fp1, Fp2, AF7, } \\
\text { AF8, PO7, PO8, } \\
\text { O1, O2 }\end{array}$ & $\begin{array}{c}\text { Custom locations } \\
\text { over the visual } \\
\text { cortex }\end{array}$ \\
\hline $\begin{array}{r}\text { Additional } \\
\text { Sensors: }\end{array}$ & 9-Axis IMU & 9-Axis IMU & 9-Axis IMU & None \\
\hline Resolution: & 24bits & 24bits & 24bits & - \\
\hline $\begin{array}{r}\text { Sampling } \\
\text { rate: }\end{array}$ & $256 \mathrm{~Hz}$ & $256 \mathrm{~Hz}$ & $256 \mathrm{~Hz}$ & - \\
\hline Protocol: & Bluetooth 2.1 & Bluetooth 2.1 & Bluetooth 2.1 & BLE 4.0 \\
\hline Battery: & $>3 \mathrm{~h}$ & $>8 \mathrm{~h}$ & $>8 \mathrm{~h}$ & 240 mAh: $8 \mathrm{H}$ \\
\hline
\end{tabular}

B WIRELESS EMG SENSORS

Table 9. Wireless EMG Sensors

\begin{tabular}{|c|c|c|c|c|}
\hline Device: & 1. MYO Band [41] & $\begin{array}{c}\text { 2. Gforce } \\
\text { OyMotion Band }\end{array}$ & 3. DTing One [15] & $\begin{array}{l}\text { 4. MuscleBANBE } \\
\text { [29] }\end{array}$ \\
\hline Channels: & 8 & 8 & 8 & 1 \\
\hline $\begin{array}{r}\text { Additional } \\
\text { Sensors: }\end{array}$ & 9-Axis IMU, 13 bit & $\begin{array}{c}\text { 9-Axis, } \\
\text { Accelerometer, } \\
\text { Gyroscope, } \\
\text { Magnetometer } \\
\text { 50Hz, 16bit }\end{array}$ & $\begin{array}{l}\text { Accelerometer, } \\
\text { Gyroscope: } 200 \mathrm{~Hz} \text {, } \\
\text { 16bits }\end{array}$ & $\begin{array}{l}\text { Accelerometer: } \\
\text { 14bits; } \\
\text { Magnetometer: } \\
\text { 16bits }\end{array}$ \\
\hline Resolution: & 16bits & 8bits & 12bits & 12bits \\
\hline $\begin{array}{r}\text { Sampling } \\
\text { rate: }\end{array}$ & $200 \mathrm{~Hz}$ & $1000 \mathrm{~Hz}$ & $1000 \mathrm{~Hz}$ & $1000 \mathrm{~Hz}$ \\
\hline Protocol: & BLE & BLE4.1 & BLE 5.0, Wi-Fi & BLE \\
\hline Battery: & $2 \times 260 \mathrm{mAh}$ & $200 \mathrm{mAh}$ & - & $155 \mathrm{mAh}: 8 \mathrm{~h}$ \\
\hline
\end{tabular}




\section{A QUESTIONNAIRE ON SOCIAL ACCEPTANCE OF NOVEL USER INTERFACES}

Below we list the questions we asked to 93 participants using Google Forms together with the available answers and the number of the participants selected each answer. We also report mean $(\bar{M})$ and standard deviation values $(\sigma)$ in either a 5-point scale ranged from -2 to $2(-2,-1,0,1,2)$ for 5 -option questions or a 3 -point scale ranged from -1 to $1(-1,0,1)$ for 3 -option questions.

\section{Introduction}

Question 1: Experience with Augmented Reality (AR) and Virtual Reality (VR), and novel HCI interfaces.

Available answers and spread of the 93 responses for $\operatorname{AR}(\bar{M}=0.85) / \mathrm{VR}(\bar{M}=0.85) /$ Novel HCI $(\bar{M}$ $=0.11)$ :

(1) It is the first time I hear about this.

(AR: 4 / VR: 0 / Novel HCI: 38)

(2) I have heard about/seen that.

(3) I have some experience with that.

(4) I do use this technology on regular basis.

(5) I do research in that field / I develop solutions in this area.

\section{Acceptance of Brain computer interfaces}

Question 2: If we assume that BCI, as an alternative to classical input devices, will be adopted by community, what will be your attitude towards it? Available answers and spread of the 93 responses $(\bar{M}=0.90, \sigma=1.16)$ :

(1) It is unacceptable for me to use this in public (as well as at home).

(2) It is unacceptable for me to use this at home.

(3) Not sure. $\quad 7$

(4) It is OK for me to use this at home.

(5) It is OK for me to use this in public (as well as at home).

Question 3: If you have chosen a positive answer in the previous question, tell us how enthusiastic you would be about this tech? Available answers and spread of the 80 responses $(\bar{M}=0.91, \sigma=0.51)$ :

(1) I will be the first one to buy it!

(2) I will get it once it becomes mainstream.

(3) I will wait till someone gifts me the thing.

Question 4: Will you disapprove, accept or appreciate if people around you would use BCI devices? Available answers and spread of the 93 responses $(\bar{M}=1.19, \sigma=1.10)$ :

(1) It is unacceptable for me if somebody uses at my home (as well as in public). 3

(2) It is unacceptable for me if somebody uses this in public.

(3) Not sure. $\quad 18$

(4) It is OK for me if somebody uses this in public (but not at my home).

(5) It is OK for me if somebody uses at my home (as well as in public).

\section{Acceptance of Muscle-powered interfaces}

Question 5: If we assume that gesture-based inputs, will become popular, what will be your attitude towards it? Available answers and spread of the 93 responses $(\bar{M}=1.18, \sigma=1.04)$ :

(1) It is unacceptable for me to use this in public (as well as at home).

(2) It is unacceptable for me to use this at home.

(3) Not sure. 13 
(4) It is OK for me to use this at home.

(5) It is OK for me to use this in public (as well as at home).

Question 6: If you have chosen a positive answer in the previous question, tell us how enthusiastic you would be about getting a sEMG armband and actively gesticulating? Available answers and spread of the 82 responses $(\bar{M}=0.98, \sigma=0.57)$ :

(1) I will be the first one to buy it! 12

(2) I will get it once it becomes mainstream. 56

(3) I will wait till someone gifts me the thing.

Question 7: Will you disapprove, accept or appreciate if people around you would moderately intensively make gestures midair? Available answers and spread of the 93 responses $(\bar{M}=1.19, \sigma=1.09)$ :

(1) It is unacceptable for me if somebody uses at my home (as well as in public).

\section{1}

(2) It is unacceptable for me if somebody uses this in public.

(3) Not sure. 17

(4) It is OK for me if somebody uses this in public (but not at my home). 12

(5) It is OK for me if somebody uses at my home (as well as in public).

\section{Acceptance of Silent Speech interfaces}

Question 8: If we assume that SSI, will become popular, what will be your attitude towards it? Available answers and spread of the 93 responses $(\bar{M}=0.44, \sigma=1.39)$ :

(1) It is unacceptable for me to use this in public (as well as at home).

(2) It is unacceptable for me to use this at home.

(3) Not sure.

(4) It is OK for me to use this at home.

(5) It is OK for me to use this in public (as well as at home).

Question 9: If you have chosen a positive answer in the previous question, tell us how enthusiastic you would be about getting a SSI wearable interface? Available answers and spread of the 70 responses from the participants that gave a positive answer in Question $8(\bar{M}=0.59, \sigma=0.60)$ :

(1) I will be the first one to buy it!

(2) I will get it once it becomes mainstream.

(3) I will wait till someone gifts me the thing.

Question 10: Will you disapprove, accept or appreciate if people around you would wear those SSIs and whisper things to themselves? Available answers and spread of the 93 responses $(\bar{M}=0.93, \sigma=$ 1.23):

(1) It is unacceptable for me if somebody uses at my home (as well as in public). 4

(2) It is unacceptable for me if somebody uses this in public. 9

(3) Not sure. 21

(4) It is OK for me if somebody uses this in public (but not at my home). 3

(5) It is OK for me if somebody uses at my home (as well as in public).

\section{Acceptance of Gaze-controlled interfaces}

Question 11: If we assume that Gaze-control interfaces, will become popular, what will be your attitude towards it? Available answers and spread of the 93 responses $(\bar{M}=1.17, \sigma=1.35)$ :

(1) It is unacceptable for me to use this in public (as well as at home).

(2) It is unacceptable for me to use this at home.

(3) Not sure. 
(4) It is OK for me to use this at home.

(5) It is OK for me to use this in public (as well as at home).

Question 12: If you have chosen a positive answer in the previous question, tell us how enthusiastic you would be about getting one of eye-tracking glasses? Available answers and spread of the 81 responses from the participants that gave a positive answer in Question $11(\bar{M}=1.06, \sigma=0.70)$ :

(1) I will be the first one to buy it!

(2) I will get it once it becomes mainstream.

(3) I will wait till someone gifts me the thing.

Question 13: Will you disapprove, accept or appreciate if people around you would wear eye-tracking glasses? Available answers and spread of the 93 responses $(\bar{M}=1.43, \sigma=1.05)$ :

(1) It is unacceptable for me if somebody uses at my home (as well as in public).

(2) It is unacceptable for me if somebody uses this in public.

(3) Not sure.

(4) It is OK for me if somebody uses this in public (but not at my home).

(5) It is OK for me if somebody uses at my home (as well as in public).

\section{ACKNOWLEDGMENTS}

The authors thank the anonymous reviewers for constructive feedback throughout the review cycle and professor Rosa H. M. Chan (City University of Hong Kong) for her fruitful suggestions. We also express our gratitude toward the participants of the survey (see Section 6), who helped us outline the degree of acceptance of novel ExG-based interfaces.

\section{REFERENCES}

[1] [n.d.]. 13E200 MyoBock Electrode. Retrieved July 8, 2019 from https://professionals.ottobock.com.au/Products/ Prosthetics/Prosthetics-Upper-Limb/Adult-Terminal-Devices/13E200-MyoBock-electrode/p/13E200.

[2] [n.d.]. Air-Dry EEG Device for Basic Brain States Estimation| Bitbrain. Retrieved February 11, 2021 from https: //www.bitbrain.com/neurotechnology-products/dry-eeg/air.

[3] [n.d.]. Athos Coaching System. Retrieved November 11, 2020 from https://shop.liveathos.com/.

[4] [n.d.]. Auris. Retrieved June 22, 2019 from https://www.cognionics.net/auris.

[5] [n.d.]. B-Alert X24. Retrieved June 22, 2019 from https://www.advancedbrainmonitoring.com/xseries/x24/.

[6] [n.d.]. BR32S. Wireless EEG System for Brain-Computer-Interface. Retrieved Jun 22, 2019 from http://www.physiotech.co.jp/pdf/br/br32s.pdf.

[7] [n.d.]. BR8 PLUS. Retrieved June 22, 2019 from http://www.physio-tech.co.jp/products/br/br8_plus.html.

[8] [n.d.]. cEEGrid. Retrieved June 22, 2019 from http://ceegrid.com/home/.

[9] [n.d.]. Dev Kit EEG Headband with Dry Electrodes. Retrieved June 22, 2019 from https://www.cognionics.net/copyof-dev-kit.

[10] [n.d.]. Dev Kit Specifications. Retrieved February 11, 2021 from https://www.next-mind.com/product/specs/.

[11] [n.d.]. Diadem-Wearable Dry-EEG Headset. Retrieved February 11, 2021 from https://www.bitbrain.com/ neurotechnology-products/dry-eeg/diadem.

[12] [n.d.]. DSI 24-Wearable Sensing. Retrieved June 22, 2019 from https://wearablesensing.com/products/dsi-24/.

[13] [n.d.]. DSI 7-Wearable Sensing. Retrieved June 22, 2019 from https://wearablesensing.com/products/dsi-7-wirelessdry-eeg-headset/.

[14] [n.d.]. DSI-VR300-NEUROSPEC AG Research Neurosciences. Retrieved June 22, 2019 from https://www.neurospec. com/Products/Details/1077/dsi-vr300.

[15] [n.d.]. DTing ONE-DTing. Retrieved June 24, 2019 from http://www.dtingsmart.com/dting-one.

[16] [n.d.]. EMOTIV EPOC+ 14 Channel Mobile EEG-Emotiv. Retrieved June 22, 2019 from https://www.emotiv.com/ product/emotiv-epoc-14-channel-mobile-eeg/.

[17] [n.d.]. EMOTIV Insight 5 Channel Mobile EEG-Emotiv. Retrieved June 22, 2019 from https://www.emotiv.com/ product/emotiv-insight-5-channel-mobile-eeg/.

[18] [n.d.]. EPOC Flex Gel Sensor Kit-Emotiv. Retrieved June 22, 2019 from https://www.emotiv.com/product/epoc-flexgel-sensor-kit/. 
[19] [n.d.]. EPOC Flex Saline Sensor Kit-Emotiv. Retrieved June 22, 2019 from https://www.emotiv.com/product/epocflex-saline-sensor-kit/.

[20] [n.d.]. Gabe Newell Says Brain-Computer Interface Tech Will Allow Video Games Far Beyond What Human 'Meat Peripherals' Can Comprehend. Retrieved February 11, 2021 from https://www.tvnz.co.nz/onenews/new-zealand/gabe-newell-says-brain-computer-interface-tech-allow-video-games-far-beyond-humanmeat-peripherals-can-comprehend.

[21] [n.d.]. Galea. Retrieved February 11, 2021 from https://galea.co/.

[22] [n.d.]. g.Nautilus-g.tec's Wireless EEG System with Active Electrodes. Retrieved June 222019 from http://www.gtec. at/Products/Hardware-and-Accessories/g.Nautilus-Specs-Features.

[23] [n.d.]. Hero-Dry-EEG system for Motor Cortex monitoring. Retrieved February 11, 2021 https://www.bitbrain.com/ neurotechnology-products/dry-eeg/hero.

[24] [n.d.]. List of Interface Bit Rates. Retrieved July 18, 2019 from https://en.wikipedia.org/wiki/List_of_interface_bit_ rates.

[25] [n.d.]. LooxidVR: Make Your Research Limitless. Retrieved June 222019 from https://looxidlabs.com/looxidvr/.

[26] [n.d.]. Mindo-64 Coral. Retrieved June 22, 2019 from http://www.mindo.com.tw/en/goods.php?act=view\&no=18.

[27] [n.d.]. MINT EEG Acquisition System: JellyFish 1.0. Retrieved February 11, 2021 from https://github.com/UBCMint/ MINT_NTX2020_FixedChallenge.

[28] [n.d.]. Mobile-128 Wireless High Density EEG. Retrieved June 22, 2019 from https://www.cognionics.net/mobile-128.

[29] [n.d.]. MuscleBANBE Data Sheet. Retrieved October 15, 2020 from https://biosignalsplux.com/downloads/docs/ datasheets/muscleBAN_Datasheet.pdf.

[30] [n.d.]. Muse 2: Brain Sensing Headband-Technology Enhanced Meditation. Retrieved June 22, 2019 from https:// choosemuse.com/muse- $2 /$.

[31] [n.d.]. Muse The Brain Sensing Headband-Technology Enhanced Meditation. Retrieved June 22, 2019 from https: //choosemuse.com/muse/.

[32] [n.d.]. OpenBCI-Open Source Biosensing Tools (EEG, EMG, EKG, and more). Retrieved June 22, 2019 from https: //openbci.com/.

[33] [n.d.]. OYMotion. Retrieved June 24, 2019 https://oymotion.github.io/.

[34] [n.d.]. Product-ZETO INC. Retrieved June 28, 2019 https://zeto-inc.com/device/.

[35] [n.d.]. Project EMGeyboard. Github. Retrieved February 11, 2021 from https://github.com/NTX-McGill/NeuroTechXMcGill-2020.

[36] [n.d.]. Quick-30 Wireless Dry EEG Headset with Dry Electrodes. Retrieved June 22, 2019 from https://www. cognionics.net/quick-30.

[37] [n.d.]. Research Tools. Retrieved June 22, 2019 from https://store.neurosky.com/products/mindset-research-tools.

[38] [n.d.]. Smarting Device-High-end fully Mobile EEG devices. Retrieved June 22, 2019 from https://mbraintrain.com/ smarting/.

[39] [n.d.]. Tobii Pro Glasses 2-Product Description. Retrieved July 5, 2019 from https://www.tobiipro.com/siteassets/ tobii-pro/product-descriptions/tobii-pro-glasses-2-product-description.pdf.

[40] [n.d.]. Typing. Retrieved July 18, 2019 from https://en.wikipedia.org/wiki/Typing.

[41] [n.d.]. Welcome to Myo Support. Retrieved June 24, 2019 from https://support.getmyo.com/hc/en-us.

[42] Tobii AB. 2019. Tobii: The World Leader in Eye Tracking. Retrieved October 17, 2019 from https://www.tobii.com/.

[43] Gizem Acar, Ozberk Ozturk, Ata Jedari Golparvar, Tamador Alkhidir Elboshra, Karl Bühringer, and Murat Kaya Yapici. 2019. Wearable and flexible textile electrodes for biopotential signal monitoring: A review. Electronics 8, 5 (2019). https://doi.org/10.3390/electronics8050479

[44] Mahesh Kumar Adimulam and M. B. Srinivas. 2016. Modeling of EXG (ECG, EMG and EEG) non-idealities using MATLAB. In Proceedings of the 2016 9th International Congress on Image and Signal Processing, BioMedical Engineering and Informatics (CISP-BMEI'16). IEEE, 1584-1589.

[45] Sunggeun Ahn, Jeongmin Son, Sangyoon Lee, and Geehyuk Lee. 2020. Verge-it: Gaze interaction for a binocular headworn display using modulated disparity vergence eye movement. In Extended Abstracts of the 2020 CHI Conference on Human Factors in Computing Systems (CHI EA'20). Association for Computing Machinery, New York, NY, 1-7. https://doi.org/10.1145/3334480.3382908

[46] S. Aleksandar. 2019. How Much Time Do People Spend on Social Media in 2019? Retrieved October 21, 2019 from https://techjury.net/blog/time-spent-on-social-media/.

[47] Mohammed Alnemari. 2017. Integration of a Low Cost EEG Headset with The Internet of Thing Framework. Ph.D. Dissertation. UC Irvine.

[48] Leopoldo Angrisani, Pasquale Arpaia, Antonio Esposito, and Nicola Moccaldi. 2020. A wearable brain-computer interface instrument for augmented reality-based inspection in industry 4.0. IEEE Trans. Instrum. Meas. 69, 4 (2020), $1530-1539$. 
[49] Inc. Apple. 2019. AR Kit 3-Apple Developers. Retrieved October 17, 2019 from https://developer.apple.com/ augmented-reality/.

[50] Kikuo Asai, Yuji Y. Sugimoto, Narong Hemmakorn, Noritaka Osawa, and Kimio Kondo. 2004. Language-support system using character recognition. In Proceedings of the 2004 ACM SIGGRAPH International Conference on Virtual Reality Continuum and Its Applications in Industry (VRCAI'04). Association for Computing Machinery, New York, NY, 173-179. https://doi.org/10.1145/1044588.1044623

[51] Ilhan Aslan, Andreas Uhl, Alexander Meschtscherjakov, and Manfred Tscheligi. 2014. Mid-air authentication gestures: An exploration of authentication based on palm and finger motions. In Proceedings of the 16th International Conference on Multimodal Interaction (ICMI'14). Association for Computing Machinery, New York, NY, 311-318. https://doi.org/10.1145/2663204.2663246

[52] Ilhan Aslan, Andreas Uhl, Alexander Meschtscherjakov, and Manfred Tscheligi. 2016. Design and exploration of midair authentication gestures. ACM Trans. Interact. Intell. Syst. 6, 3, Article 23 (Sep. 2016), 22 pages. https://doi.org/10. $1145 / 2832919$

[53] Md Moin Uddin Atique, Sakhawat Hossen Rakib, and Khondkar Siddique-e Rabbani. 2016. An electrooculogram based control system. In Proceedings of the 2016 5th International Conference on Informatics, Electronics and Vision (ICIEV'16). IEEE, 809-812.

[54] Manfredo Atzori, Matteo Cognolato, and Henning Muller. 2016. Deep learning with convolutional neural networks applied to electromyography data: A resource for the classification of movements for prosthetic hands. Front. Neurorobot. 10 (2016), 9. https://doi.org/10.3389/fnbot.2016.00009

[55] Heber Avalos-Viveros, Guillermo Molero-Castillo, Edgard Benitez-Guerrro, and Everardo Bárcenas. 2018. Towards a method for biosignals analysis as support for the design of adaptive user-interfaces. Adv. Pattern Recogn. 9 (2018), 9-19.

[56] S. Bakker and K. Niemantsverdriet. 2016. The interaction-attention continuum: Considering various levels of human attention in interaction design. Int. f. Des. 10 (2016), 1-14.

[57] Domna Banakou, Parasuram D. Hanumanthu, and Mel Slater. 2016. Virtual embodiment of white people in a black virtual body leads to a sustained reduction in their implicit racial bias. Front. Hum. Neurosci. 10 (2016), 601. https: //doi.org/10.3389/fnhum.2016.00601

[58] Javier A. Bargas-Avila and Kasper Hornbæk. 2011. Old wine in new bottles or novel challenges: A critical analysis of empirical studies of user experience. In Proceedings of the SIGCHI Conference on Human Factors in Computing Systems (CHI'11). Association for Computing Machinery, New York, NY, 2689-2698. https://doi.org/10.1145/1978942.1979336

[59] Vincent Becker, Pietro Oldrati, Liliana Barrios, and Gábor Sörös. 2018. Touchsense: Classifying finger touches and measuring their force with an electromyography armband. In Proceedings of the 2018 ACM International Symposium on Wearable Computers. ACM, 1-8.

[60] Ilyasse Belkacem, Isabelle Pecci, Benoît Martin, and Anthony Faiola. 2019. TEXTile: Eyes-free text input on smart glasses using touch enabled textile on the forearm. In Proceedings of the Human-Computer Interaction (INTERACT'19), David Lamas, Fernando Loizides, Lennart Nacke, Helen Petrie, Marco Winckler, and Panayiotis Zaphiris (Eds.). Springer International Publishing, Cham, 351-371.

[61] Carlos Bermejo, Tristan Braud, Ji Yang, Shayan Mirjafari, Bowen Shi, Yu Xiao, and Pan Hui. 2020. VIMES: A wearable memory assistance system for automatic information retrieval. In Proceedings of the 28th ACM International Conference on Multimedia. 3191-3200.

[62] Carlos Bermejo and Pan Hui. 2017. A survey on haptic technologies for mobile augmented reality. arXiv:1709.00698. Retrieved from https://arxiv.org/abs/1709.00698.

[63] Guillermo Bernal and Pattie Maes. 2017. Emotional beasts: Visually expressing emotions through avatars in VR. In Proceedings of the 2017 CHI Conference Extended Abstracts on Human Factors in Computing Systems. 2395-2402.

[64] Guillermo Bernal, Tao Yang, Abhinandan Jain, and Pattie Maes. 2018. PhysioHMD: A conformable, modular toolkit for collecting physiological data from head-mounted displays. In Proceedings of the 2018 ACM International Symposium on Wearable Computers. 160-167.

[65] BITalino. 2019. Project BITalino. Retrieved October 17, 2019 from https://bitalino.com/en/.

[66] Damien Brun, Charles Gouin-Vallerand, and Sébastien George. 2017. Augmented human mind: Case of reasoning. In Proceedings of the 2017 ACM International foint Conference on Pervasive and Ubiquitous Computing and Proceedings of the 2017 ACM International Symposium on Wearable Computers (UbiComp'17). Association for Computing Machinery, New York, NY, 717-723. https://doi.org/10.1145/3123024.3129273

[67] BTS. 2019. BTS Bioengineering. Retrieved October 17, 2019 from https://www.btsbioengineering.com/?page_id= 12786.

[68] Andreas Bulling, Daniel Roggen, and Gerhard Tröster. 2009. Wearable EOG Goggles: Eye-based Interaction in Everyday Environments. ACM.

[69] Darryl H. Burnet and Matthew D. Turner. 2017. Expanding EEG research into the clinic and classroom with consumer EEG Systems. Georgia State Undergraduate Research Conference.

ACM Transactions on Interactive Intelligent Systems, Vol. 11, No. 2, Article 10. Publication date: July 2021. 
[70] E. Cafaro, L. Saue-Fletcher, M. J. Washington, M. Bijanzadeh, A. M. Lee, E. F. Chang, A. D. Huberman, et al. 2020. Human responses to visually evoked threat. Curr. Biol. (2020).

[71] D. Chatzopoulos, C. Bermejo, Z. Huang, and P. Hui. 2017. Mobile augmented reality survey: From where we are to where we go. IEEE Access 5 (2017), 6917-6950. https://doi.org/10.1109/ACCESS.2017.2698164

[72] Aritra Chaudhuri, Anirban Dasgupta, Suvodip Chakrborty, and Aurobinda Routray. 2016. A low-cost, wearable, portable EOG recording system. In Proceedings of the 2016 International Conference on Systems in Medicine and Biology (ICSMB'16). IEEE, 102-105.

[73] C. G. Coogan and B. He. 2018. Brain-computer interface control in a virtual reality environment and applications for the internet of things. IEEE Access 6 (2018), 10840-10849.

[74] David Costa and Carlos Duarte. 2015. From one to many users and contexts: A classifier for hand and arm gestures. In Proceedings of the 20th International Conference on Intelligent User Interfaces (IUI'15). Association for Computing Machinery, New York, NY, 115-120. https://doi.org/10.1145/2678025.2701388

[75] Ulysse Côté-Allard, Gabriel Gagnon-Turcotte, François Laviolette, and Benoit Gosselin. 2019. A low-cost, wireless, 3-d-printed custom armband for semg hand gesture recognition. Sensors 19, 12 (2019), 2811.

[76] Keith M. Davis, Lauri Kangassalo, Michiel Spapé, and Tuukka Ruotsalo. 2020. Brainsourcing: Crowdsourcing recognition tasks via collaborative brain-computer interfacing. In Proceedings of the 2020 CHI Conference on Human Factors in Computing Systems (CHI'20). Association for Computing Machinery, New York, NY, 1-14. https://doi.org/10.1145/ 3313831.3376288

[77] Yunbin Deng, James T. Heaton, and Geoffrey S. Meltzner. 2014. Towards a practical silent speech recognition system. In Proceedings of the 15th Annual Conference of the International Speech Communication Association.

[78] C. Nathan DeWall and Brad J. Bushman. 2011. Social acceptance and rejection: The sweet and the bitter. Curr. Direct. Psychol. Sci. 20, 4 (2011), 256-260.

[79] Murtaza Dhuliawala, Juyoung Lee, Junichi Shimizu, Andreas Bulling, Kai Kunze, Thad Starner, and Woontack Woo. 2016. Smooth eye movement interaction using EOG glasses. In Proceedings of the 18th ACM International Conference on Multimodal Interaction. ACM, 307-311.

[80] S. Z. Diya, R. A. Prorna, I. I. Rahman, A. B. Islam, and M. N. Islam. 2019. Applying brain-computer interface technology for evaluation of user experience in playing games. In Proceedings of the 2019 International Conference on Electrical, Computer and Communication Engineering (ECCE'19). 1-6. https://doi.org/10.1109/ECACE.2019.8679203

[81] Andreas Dünser, Lawrence Walker, Heather Horner, and Daniel Bentall. 2012. Creating interactive physics education books with augmented reality. In Proceedings of the 24th Australian Computer-Human Interaction Conference (OzCHI'12). Association for Computing Machinery, New York, NY, 107-114. https://doi.org/10.1145/2414536.2414554

[82] Ellen Dupler and Lucy E. Dunne. 2019. Effects of the textile-sensor interface on stitched strain sensor performance. In Proceedings of the 23rd International Symposium on Wearable Computers (ISWC'19). 45-53. https://doi.org/10.1145/ 3341163.3347717

[83] Boyu Fan, Xuefeng Liu, Xiang Su, Pan Hui, and Jianwei Niu. 2020. Emgauth: An emg-based smartphone unlocking system using siamese network. In Proceedings of the IEEE International Conference on Pervasive Computing and Communications (PerCom'20). IEEE, 1-10.

[84] Jacqui Fashimpaur, Kenrick Kin, and Matt Longest. 2020. PinchType: Text entry for virtual and augmented reality using comfortable thumb to fingertip pinches. In Extended Abstracts of the $2020 \mathrm{CHI}$ Conference on Human Factors in Computing Systems (CHI EA'20). Association for Computing Machinery, New York, NY, 1-7. https://doi.org/10.1145/ 3334480.3382888

[85] Mehrdad Fatourechi, Ali Bashashati, Rabab K. Ward, and Gary E. Birch. 2007. EMG and EOG artifacts in brain computer interface systems: A survey. Clin. Neurophysiol. 118, 3 (2007), 480-494.

[86] Oliver Faust, Yuki Hagiwara, Tan Jen Hong, Oh Shu Lih, and U. Rajendra Acharya. 2018. Deep learning for healthcare applications based on physiological signals: A review. Comput. Methods Progr. Biomed. 161 (2018), 1-13.

[87] Jan Fischer, Markus Neff, Dirk Freudenstein, and Dirk Bartz. 2004. Medical augmented reality based on commercial image guided surgery. In Proceedings of the Eurographics Symposium on Virtual Environments (EGVE'04). 83-86.

[88] João Freitas, Artur Ferreira, Mário Figueiredo, António Teixeira, and Miguel Sales Dias. 2014. Enhancing multimodal silent speech interfaces with feature selection. In Proceedings of the 15th Annual Conference of the International Speech Communication Association. 
[89] Yang Gao, Wei Wang, Vir V. Phoha, Wei Sun, and Zhanpeng Jin. 2019. EarEcho: Using ear canal echo for wearable authentication. Proc. ACM Interact. Mobile Wear. Ubiq. Technol. 3, 3 (2019), 1-24.

[90] Inc. Google. 2019. AR Core-Google Developers. Retrieved October 17, 2019 from https://developers.google.com/ar/.

[91] Qiong Gui, Maria V. Ruiz-Blondet, Sarah Laszlo, and Zhanpeng Jin. 2019. A survey on brain biometrics. ACM Comput. Surv. 51, 6, Article 112 (Feb. 2019), 38 pages. https://doi.org/10.1145/3230632

[92] Faizan Haque, Mathieu Nancel, and Daniel Vogel. 2015. Myopoint: Pointing and clicking using forearm mounted electromyography and inertial motion sensors. In Proceedings of the 33rd Annual ACM Conference on Human Factors in Computing Systems. ACM, 3653-3656.

[93] Takuto Hayashi, Masaru Ohkubo, Sho Sakurai, Koichi Hirota, and Takuya Nojima. 2019. Towards making kinetic garments based on conductive fabric and smart hair. In Proceedings of the 23rd International Symposium on Wearable Computers (ISWC'19). ACM, New York, NY, 89-90. https://doi.org/10.1145/3341163.3347733

[94] Suzana Herculano-Houzel. 2009. The human brain in numbers: A linearly scaled-up primate brain. Front. Hum. Neurosci. 3 (2009), 31.

[95] Arne Holst. [n.d.]. Number of mobile devices worldwide 2019-2023. Retrieved July 6, 2019 from https://www.statista. com/statistics/245501/multiple-mobile-device-ownership-worldwide/.

[96] Keum-Shik Hong and Muhammad Jawad Khan. 2017. Hybrid brain-computer interface techniques for improved classification accuracy and increased number of commands: A review. Front. Neurorobot. 11 (2017), 35. https://doi. org/10.3389/fnbot.2017.00035

[97] Zhanpeng Huang, Weikai Li, and Pan Hui. 2015. Ubii: Towards seamless interaction between digital and physical worlds. In Proceedings of the 23rd ACM International Conference on Multimedia (MM'15). Association for Computing Machinery, New York, NY, 341-350. https://doi.org/10.1145/2733373.2806266

[98] Hyunjin Yoon, Sang-Wook Park, Yong-Kwi Lee, and Jong-Hyun Jang. 2013. Emotion recognition of serious game players using a simple brain computer interface. In Proceedings of the 2013 International Conference on ICT Convergence (ICTC'13). 783-786. https://doi.org/10.1109/ICTC.2013.6675478

[99] Awesome Inc. 2019. Advancer Technologies. Retrieved October 17, 2019 from http://www.advancertechnologies. $\mathrm{com} /$.

[100] Google Inc. 2019. Glass Project: Glass Enterprise Edition. Retrieved October 21, 2019 from https://www.google.com/ glass/start/.

[101] Delsys Incorporated. 2019. Trigno Avanti Platform. Retrieved October 17, 2019 from https://www.delsys.com/trigno/.

[102] IMEC INT. 2019. Eye-Tracking Glasses: Eye-Tracking with EOG. Retrieved October 17, 2019 from https://www.imecint.com/en/eog.

[103] Shoya Ishimaru, Kai Kunze, Yuji Uema, Koichi Kise, Masahiko Inami, and Katsuma Tanaka. 2014. Smarter eyewear: Using commercial EOG glasses for activity recognition. In Proceedings of the 2014 ACM International foint Conference on Pervasive and Ubiquitous Computing: Adjunct Publication. ACM, 239-242.

[104] Krzysztof Izdebski, Anderson Souza Oliveira, Bryan R. Schlink, Petr Legkov, Silke Kärcher, W. David Hairston, Daniel P. Ferris, and Peter König. 2016. Usability of EEG systems: User experience study. In Proceedings of the 9th ACM International Conference on PErvasive Technologies Related to Assistive Environments (PETRA'16). Association for Computing Machinery, New York, NY. https://doi.org/10.1145/2910674.2910714

[105] Alice F. Jackson and Donald J. Bolger. 2014. The neurophysiological bases of EEG and EEG measurement: A review for the rest of us. Psychophysiology 51, 11 (2014), 1061-1071.

[106] Robert J. K. Jacob, Audrey Girouard, Leanne M. Hirshfield, Michael S. Horn, Orit Shaer, Erin Treacy Solovey, and Jamie Zigelbaum. 2008. Reality-based interaction: A framework for post-WIMP interfaces. In Proceedings of the SIGCHI Conference on Human Factors in Computing Systems (CHI'08). Association for Computing Machinery, New York, NY, 201-210. https://doi.org/10.1145/1357054.1357089

[107] Jhilmil Jain, Arnold Lund, and Dennis Wixon. 2011. The future of natural user interfaces. In CHI'11 Extended Abstracts on Human Factors in Computing Systems. ACM, 211-214.

[108] Lars-Erik Janlert. 2014. The ubiquitous button. Interactions 21, 3 (May 2014), 26-33. https://doi.org/10.1145/2592234

[109] Jay Jantz, Adam Molnar, and Ramses Alcaide. 2017. A brain-computer interface for extended reality interfaces. In ACM SIGGRAPH 2017 VR Village. 1-2.

[110] Charles Jorgensen and Sorin Dusan. 2010. Speech interfaces based upon surface electromyography. Speech Commun. 52, 4 (2010), 354-366.

[111] Arnav Kapur, Shreyas Kapur, and Pattie Maes. 2018. Alterego: A personalized wearable silent speech interface. In Proceedings of the 23rd International Conference on Intelligent User Interfaces. ACM, 43-53.

[112] Moritz Kassner, William Patera, and Andreas Bulling. 2014. Pupil: An open source platform for pervasive eye tracking and mobile gaze-based interaction. In Proceedings of the 2014 ACM International foint Conference on Pervasive and Ubiquitous Computing: Adjunct Publication. ACM, 1151-1160. 
[113] Yufeng Ke, Pengxiao Liu, Xingwei An, Xizi Song, and Dong Ming. 2020. An online SSVEP-BCI system in an optical see-through augmented reality environment. F. Neur. Eng. 17, 1 (2020), 016066.

[114] Dominik Kirst and Andreas Bulling. 2016. On the verge: Voluntary convergences for accurate and precise timing of gaze input. In Proceedings of the 2016 CHI Conference Extended Abstracts on Human Factors in Computing Systems (CHI EA'16). Association for Computing Machinery, New York, NY, 1519-1525. https://doi.org/10.1145/2851581.2892307

[115] Scott R. Klemmer, Björn Hartmann, and Leila Takayama. 2006. How bodies matter: Five themes for interaction design. In Proceedings of the 6th Conference on Designing Interactive Systems (DIS'06). 140-149. https://doi.org/10.1145/ 1142405.1142429

[116] Pascal Knierim, Thomas Kosch, Johannes Groschopp, and Albrecht Schmidt. 2020. Opportunities and challenges of text input in portable virtual reality. In Extended Abstracts of the $2020 \mathrm{CHI}$ Conference on Human Factors in Computing Systems (CHI EA'20). Association for Computing Machinery, New York, NY, 1-8. https://doi.org/10.1145/3334480. 3382920

[117] Martin Kocur, Valentin Schwind, and Niels Henze. 2019. Utilizing the proteus effect to improve interactions using full-body avatars in virtual reality. In Mensch und Computer 2019-Workshopband (2019).

[118] Nataliya Kosmyna, Caitlin Morris, Utkarsh Sarawgi, Thanh Nguyen, and Pattie Maes. 2019. AttentivU: A wearable pair of EEG and EOG glasses for real-time physiological processing. In Proceedings of the 2019 IEEE 16th International Conference on Wearable and Implantable Body Sensor Networks (BSN'19). IEEE, 1-4.

[119] Panagiotis Kourtesis, Danai Korre, Simona Collina, Leonidas A. A. Doumas, and Sarah E. MacPherson. 2020. Guidelines for the development of immersive virtual reality software for cognitive neuroscience and neuropsychology: The development of virtual reality everyday assessment lab (VR-EAL), a neuropsychological test battery in immersive virtual reality. Front. Comput. Sci. 1 (2020), 12. https://doi.org/10.3389/fcomp.2019.00012

[120] Hiroki Kurosawa, Daisuke Sakamoto, and Tetsuo Ono. 2018. MyoTilt: A target selection method for smartwatches using the tilting operation and electromyography. In Proceedings of the 20th International Conference on HumanComputer Interaction with Mobile Devices and Services (MobileHCI'18). Association for Computing Machinery, New York, NY, Article 43, 11 pages. https://doi.org/10.1145/3229434.3229457

[121] Young D. Kwon, Kirill A. Shatilov, Lik-Hang Lee, Serkan Kumyol, Kit-Yung Lam, Yui-Pan Yau, and Pan Hui. 2020. Myokey: Surface electromyography and inertial motion sensing-based text entry in ar. In Proceedings of the 2020 IEEE International Conference on Pervasive Computing and Communications Workshops (PerCom Workshops). IEEE, 1-4.

[122] Mikko Kytö, Barrett Ens, Thammathip Piumsomboon, Gun A. Lee, and Mark Billinghurst. 2018. Pinpointing: Precise head- and eye-based target selection for augmented reality. In Proceedings of the 2018 CHI Conference on Human Factors in Computing Systems (CHI'18). Association for Computing Machinery, New York, NY, 1-14. https://doi.org/ $10.1145 / 3173574.3173655$

[123] The McGill Physiology Virtual Lab. [n.d.]. EOG. Retrieved July 17, 2019 from https://www.medicine.mcgill.ca/physio/ vlab/Other_exps/EOG/eogintro_n.htm.

[124] Karl LaFleur, Kaitlin Cassady, Alexander Doud, Kaleb Shades, Eitan Rogin, and Bin He. 2013. Quadcopter control in three-dimensional space using a noninvasive motor imagery-based brain-computer interface. 7. Neur. Eng. 10, 4 (2013), 046003.

[125] K. Y. Lam, L. H. Lee, T. Braud, and P. Hui. 2019. M2A: A framework for visualizing information from mobile web to mobile augmented reality. In Proceedings of the 2019 IEEE International Conference on Pervasive Computing and Communications (PerCom'19). 1-10. https://doi.org/10.1109/PERCOM.2019.8767388

[126] Hong Ji Lee, Hyun Seok Kim, and Kwang Suk Park. 2013. A study on the reproducibility of biometric authentication based on electroencephalogram (EEG). In Proceedings of the 2013 th international IEEE/EMBS Conference on Neural Engineering (NER'13). IEEE, 13-16.

[127] Jaehyung Lee, Kabmun Cha, Hyungmin Kim, Junhyuk Choi, Choonghyun Kim, and Songjoo Lee. 2019. Hybrid MISSSEP paradigm for classifying left and right movement toward bci for exoskeleton control. In Proceedings of the 2019 7th International Winter Conference on Brain-Computer Interface (BCI'19). IEEE, 1-3.

[128] Lik-Hang LEE. 2019. Embodied Interaction on Constrained Interfaces. HKUST Online Thesis.

[129] Lik Hang Lee, Tristan Braud, Farshid Hassani Bijarbooneh, and Pan Hui. 2019. TiPoint: Detecting fingertip for mid-air interaction on computational resource constrained smartglasses. In Proceedings of the 23rd International Symposium on Wearable Computers (ISWC'19). ACM, New York, NY, 118-122. https://doi.org/10.1145/3341163.3347723

[130] Lik Hang Lee, Tristan Braud, Farshid Hassani Bijarbooneh, and Pan Hui. 2020. UbiPoint: Towards non-intrusive midair interaction for hardware constrained smart glasses. In Proceedings of the 11th ACM Multimedia Systems Conference (MMSys'20). Association for Computing Machinery, New York, NY, 190-201. https://doi.org/10.1145/3339825.3391870

[131] Lik-Hang Lee and Pan Hui. 2018. Interaction methods for smart glasses: A survey. IEEE Access 6 (2018), 28712-28732.

[132] Lik Hang Lee, Kit Yung Lam, Tong Li, Tristan Braud, Xiang Su, and Pan Hui. 2019. Quadmetric optimized thumb-tofinger interaction for force assisted one-handed text entry on mobile headsets. Proc. ACM Interact. Mob. Wear. Ubiq. Technol. 3, 3, Article 94 (Sep. 2019), 27 pages. https://doi.org/10.1145/3351252 
[133] L. H. Lee, K. Yung Lam, Y. P. Yau, T. Braud, and P. Hui. 2019. HIBEY: Hide the keyboard in augmented reality. In Proceedings of the 2019 IEEE International Conference on Pervasive Computing and Communications (PerCom'19). 1-10. https://doi.org/10.1109/PERCOM.2019.8767420

[134] Lik-Hang Lee, Yiming Zhu, Yui-Pan Yau, Tristan Braud, Xiang Su, and Pan Hui. 2020. One-thumb text acquisition on force-assisted miniature interfaces for mobile headsets. In Proceedings of the 2020 IEEE International Conference on Pervasive Computing and Communications (PerCom'20). 1-10.

[135] Chin-Teng Lin, Li-Wei Ko, Meng-Hsiu Chang, Jeng-Ren Duann, Jing-Ying Chen, Tung-Ping Su, and Tzyy-Ping Jung. 2010. Review of wireless and wearable electroencephalogram systems and brain-computer interfaces-a mini-review. Gerontology 56, 1 (2010), 112-119.

[136] Feng Lin, Kun Woo Cho, Chen Song, Wenyao Xu, and Zhanpeng Jin. 2018. Brain password: A secure and truly cancelable brain biometrics for smart headwear. In Proceedings of the 16th Annual International Conference on Mobile Systems, Applications, and Services. 296-309.

[137] Feng Lin, Kun Woo Cho, Chen Song, Wenyao Xu, and Zhanpeng Jin. 2018. Brain password: A secure and truly cancelable brain biometrics for smart headwear. In Proceedings of the 16th Annual International Conference on Mobile Systems, Applications, and Services. 296-309.

[138] Zhonglin Lin, Changshui Zhang, Wei Wu, and Xiaorong Gao. 2006. Frequency recognition based on canonical correlation analysis for SSVEP-based BCIs. IEEE Trans. Biomed. Eng. 53, 12 (2006), 2610-2614.

[139] Kang Ling, Haipeng Dai, Yuntang Liu, and Alex X. Liu. 2018. UltraGesture: Fine-grained gesture sensing and recognition. In Proceedings of the 2018 15th Annual IEEE International Conference on Sensing, Communication, and Networking (SECON'18). IEEE, 1-9.

[140] Ruibo Liu, Qijia Shao, Siqi Wang, Christina Ru, Devin Balkcom, and Xia Zhou. 2019. Reconstructing human joint motion with computational fabrics. Proc. ACM Interact. Mob. Wear. Ubiq. Technol. 3, 1, Article 19 (Mar. 2019), 26 pages. https://doi.org/10.1145/3314406

[141] Shanhong Liu. [n.d.]. Global Augmented/Virtual Reality Market Size 2016-2023 Statistic. Retrieved July 6, 2019 from https://www.statista.com/statistics/591181/global-augmented-virtual-reality-market-size/.

[142] Shiqing Luo, Anh Nguyen, Chen Song, Feng Lin, Wenyao Xu, and Zhisheng Yan. [n.d.]. OcuLock: Exploring human visual system for authentication in virtual reality head-mounted display.

[143] S. Vaisman M. Abuhasira and A. B. Geva. [n.d.]. Retrieved July 3, 2019 from https://developer.download.nvidia.com/ video/gputechconf/gtc/2019/presentation/s9554-fast-training-of-deep-neural-networks-using-brain-generatedlabels.pdf.

[144] Jiaxin Ma, Yu Zhang, Andrzej Cichocki, and Fumitoshi Matsuno. 2014. A novel EOG/EEG hybrid human-machine interface adopting eye movements and ERPs: Application to robot control. IEEE Trans. Biomed. Eng. 62, 3 (2014), 876-889.

[145] Zheren Ma, Brandon C. Li, and Zeyu Yan. 2016. Wearable driver drowsiness detection using electrooculography signal. In Proceedings of the 2016 IEEE Topical Conference on Wireless Sensors and Sensor Networks (WiSNet'16). IEEE, 41-43.

[146] Jory MacKay. 2019. Screen Time Stats 2019: Here's How Much You Use Your Phone during the Workday. Retrieved October 21, 2019 from https://blog.rescuetime.com/screen-time-stats-2018/.

[147] Steve Mann, Tom Furness, Yu Yuan, Jay Iorio, and Zixin Wang. 2018. All reality: Virtual, augmented, mixed (x) mediated (x, y), and multimediated reality. arXiv:1804.08386. Retrieved from https://arxiv.org/abs/1804.08386.

[148] Octavio Marin-Pardo, Athanasios Vourvopoulos, Meghan Neureither, David Saldana, Esther Jahng, and Sook-Lei Liew. 2019. Electromyography as a suitable input for virtual reality-based biofeedback in stroke rehabilitation. In Proceedings of the International Conference on Human-Computer Interaction. Springer, 274-281.

[149] Diogo Marques, Luís Carriço, Tiago Guerreiro, Alexander De Luca, Pattie Maes, Ildar Muslukhov, Ian Oakley, and Emanuel von Zezschwitz. 2014. Workshop on inconspicuous interaction. In CHI'14 Extended Abstracts on Human Factors in Computing Systems. 91-94.

[150] Santosh Mathan. 2008. FEATUREImage search at the speed of thought. Interactions 15, 4 (Jul. 2008), 76-77. https: //doi.org/10.1145/1374489.1374509

[151] Fabrice Matulic, Brian Vogel, Naoki Kimura, and Daniel Vogel. 2019. Eliciting pen-holding postures for general input with suitability for EMG armband detection. In Proceedings of the 2019 ACM International Conference on Interactive Surfaces and Spaces (ISS'19). Association for Computing Machinery, New York, NY, 89-100. https://doi.org/10.1145/ 3343055.3359720

[152] Sven Mayer, Gierad Laput, and Chris Harrison. 2020. Enhancing mobile voice assistants with worldgaze. In Proceedings of the 2020 CHI Conference on Human Factors in Computing Systems (CHI'20). Association for Computing Machinery, New York, NY, 1-10. https://doi.org/10.1145/3313831.3376479

[153] JINS MEME. 2019. JINS MEME ES: Eye Sensing. Retrieved October 17, 2019 from https://jins-meme.com/en/products/ es/. 
[154] Jonathan Mercier-Ganady, Maud Marchal, and Anatole Lécuyer. 2015. B-C-invisibility power: Introducing optical camouflage based on mental activity in augmented reality. In Proceedings of the 6th Augmented Human International Conference. 97-100. https://doi.org/10.1145/2735711.2735835

[155] Microsoft. 2019. Kinect for Windows. Retrieved October 17, 2019 from https://developer.microsoft.com/en-us/ windows/kinect.

[156] Kusuma Mohanchandra, Snehanshu Saha, and G. M. Lingaraju. 2015. EEG based brain computer interface for speech communication: Principles and applications. In Brain-Computer Interfaces. Springer, 273-293.

[157] Ali Moin, Andy Zhou, Simone Benatti, Abbas Rahimi, George Alexandrov, Alisha Menon, Senam Tamakloe, Jonathan Ting, Natasha Yamamoto, Yasser Khan, Fred Burghardt, Ana C. Arias, Luca Benini, and Jan M. Rabaey. 2019. Adaptive EMG-based hand gesture recognition using hyperdimensional computing. arXiv:1901.00234. Retrieved from http: //arxiv.org/abs/1901.00234.

[158] Calkin S. Montero, Jason Alexander, Mark T. Marshall, and Sriram Subramanian. 2010. Would you do that? understanding social acceptance of gestural interfaces. In Proceedings of the 12th International Conference on Human Computer Interaction with Mobile Devices and Services. 275-278.

[159] Inc. Motion Lab Systems. 2019. Multi-Channel Electromyography Systems. Retrieved October 17, 2019 from https: //www.motion-labs.com/.

[160] NA. 2019. Low Cost Open Source eeg Device Completely Assembled USB Interface. Retrieved October 17, 2019 from https://www.olimex.com/Products/EEG/OpenEEG/EEG-SMT/open-source-hardware.

[161] Masaki Nakanishi, Yijun Wang, Xiaogang Chen, Yu-Te Wang, Xiaorong Gao, and Tzyy-Ping Jung. 2017. Enhancing detection of SSVEPs for a high-speed brain speller using task-related component analysis. IEEE Trans. Biomed. Eng. 65, 1 (2017), 104-112.

[162] Masaki Nakanishi, Yijun Wang, Yu-Te Wang, Yasue Mitsukura, and Tzyy-Ping Jung. 2014. A high-speed brain speller using steady-state visual evoked potentials. Int. f. Neur. Syst. 24, 06 (2014), 1450019.

[163] Neuralink. 2019. Neuralink Launch Event. Retrieved October 17, 2019 from https://www.youtube.com/watch?v=rvbh3t7WVI.

[164] Neuralink. 2019. ZEPTH: Intelligent Fitness and Sport Shirts. Retrieved October 17, 2019 from https://www. xiaomiyoupin.com/detail?gid=108157.

[165] Trung-Hau Nguyen and Wan-Young Chung. 2018. A Single-Channel SSVEP-Based BCI speller using deep learning. IEEE Access 7 (2018), 1752-1763.

[166] Arinobu Niijima, Takashi Isezaki, Ryosuke Aoki, Tomoki Watanabe, and Tomohiro Yamada. 2018. Biceps fatigue estimation with an E-Textile headband. In Proceedings of the 2018 ACM International Symposium on Wearable Computers (ISWC'18). Association for Computing Machinery, New York, NY, 222-223. https://doi.org/10.1145/3267242.3267281

[167] James J. S. Norton, Jessica Mullins, Birgit E. Alitz, and Timothy Bretl. 2018. The performance of 9-11-year-old children using an SSVEP-based BCI for target selection. F. Neur. Eng. 15, 5 (2018), 056012.

[168] Amin Nourmohammadi, Mohammad Jafari, and Thorsten O. Zander. 2018. A survey on unmanned aerial vehicle remote control using brain-computer interface. IEEE Trans. Hum.-Mach. Syst. 48, 4 (2018), 337-348.

[169] Michel Obbink, Hayrettin Gürkök, Danny Plass-Oude Bos, Gido Hakvoort, Mannes Poel, and Anton Nijholt. 2012. Social interaction in a cooperative brain-computer interface game. In Intelligent Technologies for Interactive Entertainment, Antonio Camurri and Cristina Costa (Eds.). Springer, Berlin, 183-192.

[170] OpenEEG. 2019. Retrieved October 17, 2019 from http://openeeg.sourceforge.net/doc/index.html.

[171] Mohammadreza Asghari Oskoei and Huosheng Hu. 2007. Myoelectric control systems survey. Biomed. Sign. Process. Contr. 2, 4 (2007), 275-294.

[172] Kseniia Palin, Anna Feit, Sunjun Kim, Per Ola Kristensson, and Antti Oulasvirta. 2019. How do people type on mobile devices? observations from a study with 37,000 volunteers.. In Proceedings of 21st International Conference on Human-Computer Interaction with Mobile Devices and Services (MobileHCI'19).

[173] Shijia Pan, Carlos Ruiz, Jun Han, Adeola Bannis, Patrick Tague, Hae Young Noh, and Pei Zhang. 2018. UniverSense: IoT device pairing through heterogeneous sensing signals. In Proceedings of the 19th International Workshop on Mobile Computing Systems \& Applications (HotMobile'18). Association for Computing Machinery, New York, NY, 55-60. https://doi.org/10.1145/3177102.3177108

[174] Nelusa Pathmanathan, Michael Becher, Nils Rodrigues, Guido Reina, Thomas Ertl, Daniel Weiskopf, and Michael Sedlmair. 2020. Eye vs. head: Comparing gaze methods for interaction in augmented reality. In Proceedings of the ACM Symposium on Eye Tracking Research and Applications (ETRA'20 Short Papers). Association for Computing Machinery, New York, NY, Article 50, 5 pages. https://doi.org/10.1145/3379156.3391829

[175] Ken Pfeuffer, Matthias J. Geiger, Sarah Prange, Lukas Mecke, Daniel Buschek, and Florian Alt. 2019. Behavioural biometrics in vr: Identifying people from body motion and relations in virtual reality. In Proceedings of the $2019 \mathrm{CHI}$ Conference on Human Factors in Computing Systems. 1-12. 
[176] Esteban J. Pino, Yerko Arias, and Pablo Aqueveque. 2018. Wearable EMG shirt for upper limb training. In Proceedings of the 2018 40th Annual International Conference of the IEEE Engineering in Medicine and Biology Society (EMBC'18). IEEE, 4406-4409.

[177] Leigh Ellen Potter and Alexandra Thompson. 2019. New and emerging technology: Ownership and adoption. In Proceedings of the 2019 on Computers and People Research Conference. 57-66.

[178] Dominic Potts, Kate Loveys, HyunYoung Ha, Shaoyan Huang, Mark Billinghurst, and Elizabeth Broadbent. 2019. ZenG: AR neurofeedback for meditative mixed reality. In Proceedings of the 2019 on Creativity and Cognition. ACM, 583-590.

[179] Jane Prophet, Yong Ming Kow, and Mark Hurry. 2018. Small trees, big data: Augmented reality model of air quality data via the chinese art of "Artificial" tray planting. In ACM SIGGRAPH 2018 Posters. Article 16, 2 pages. https://doi. org/10.1145/3230744.3230753

[180] Felix Putze. 2019. Methods and tools for using BCI with augmented and virtual reality. In Brain Art. Springer, $433-446$.

[181] Thea Radüntz. 2018. Signal quality evaluation of emerging EEG devices. Front. Physiol. 9 (2018), 98.

[182] Rabie A. Ramadan, S. Refat, Marwa A. Elshahed, and Rasha A. Ali. 2015. Basics of brain computer interface. In Brain-Computer Interfaces. Springer, 31-50.

[183] Grigory Rashkov, Anatoly Bobe, Dmitry Fastovets, and Maria Komarova. 2019. Natural image reconstruction from brain waves: A novel visual BCI system with native feedback. https://doi.org/10.1101/787101

[184] Elena Ratti, Shani Waninger, Chris Berka, Giulio Ruffini, and Ajay Verma. 2017. Comparison of medical and consumer wireless EEG systems for use in clinical trials. Front. Hum. Neurosci. 11 (2017), 398.

[185] Charlotte M. Reed and Nathaniel I. Durlach. 1998. Note on information transfer rates in human communication. Presence 7, 5 (1998), 509-518.

[186] Aya Rezeika, Mihaly Benda, Piotr Stawicki, Felix Gembler, Abdul Saboor, and Ivan Volosyak. 2018. Brain-computer interface spellers: A review. Brain Sci. 8, 4 (2018), 57.

[187] Michael Rietzler, Gabriel Haas, Thomas Dreja, Florian Geiselhart, and Enrico Rukzio. 2019. Virtual muscle force: Communicating kinesthetic forces through pseudo-haptic feedback and muscle input. In Proceedings of the 32nd Annual ACM Symposium on User Interface Software and Technology (UIST'19). Association for Computing Machinery, New York, NY, 913-922. https://doi.org/10.1145/3332165.3347871

[188] Jaime Ruiz and Daniel Vogel. 2015. Soft-constraints to reduce legacy and performance bias to elicit whole-body gestures with low arm fatigue. In Proceedings of the 33rd Annual ACM Conference on Human Factors in Computing Systems (CHI'15). Association for Computing Machinery, New York, NY, 3347-3350. https://doi.org/10.1145/2702123. 2702583

[189] PLUX Wireless Biosignals S.A. 2019. Bio-signal PLUX. Retrieved October 17, 2019 from https://plux.info/.

[190] Arun Sahayadhas, Kenneth Sundaraj, and Murugappan Murugappan. 2012. Detecting driver drowsiness based on sensors: A review. Sensors 12, 12 (2012), 16937-16953.

[191] Christina Schneegass, Thomas Kosch, Andrea Baumann, Marius Rusu, Mariam Hassib, and Heinrich Hussmann. 2020. BrainCoDe: Electroencephalography-based comprehension detection during reading and listening. In Proceedings of the 2020 CHI Conference on Human Factors in Computing Systems (CHI'20). Association for Computing Machinery, New York, NY, 1-13. https://doi.org/10.1145/3313831.3376707

[192] Tanja Schultz, Michael Wand, Thomas Hueber, Dean J. Krusienski, Christian Herff, and Jonathan S. Brumberg. 2017. Biosignal-based spoken communication: A survey. IEEE/ACM Trans. Aud. Speech Lang. Process. 25, 12 (2017), 22572271.

[193] Valentin Schwind, Jens Reinhardt, Rufat Rzayev, Niels Henze, and Katrin Wolf. 2018. On the need for standardized methods to study the social acceptability of emerging technologies. In CHI'18 Workshop on (Un) Acceptable.

[194] Claude Elwood Shannon. 1948. A mathematical theory of communication. Bell Syst. Techn. 7. 27, 3 (1948), $379-423$.

[195] Adwait Sharma, Joan Sol Roo, and Jürgen Steimle. 2019. Grasping microgestures: Eliciting single-hand microgestures for handheld objects. In Proceedings of the 2019 CHI Conference on Human Factors in Computing Systems. Article Paper 402, 13 pages.

[196] Kirill A. Shatilov, Dimitris Chatzopoulos, Alex Wong Tat Hang, and Pan Hui. 2019. Using deep learning and mobile offloading to control a 3d-printed prosthetic hand. Proc. ACM Interact. Mob. Wear. Ubiq. Technol. 3, 3, Article 102 (Sep. 2019), 19 pages. https://doi.org/10.1145/3351260

[197] Hakim Si-Mohammed, Jimmy Petit, Camille Jeunet, Ferran Argelaguet, Fabien Spindler, Andéol Evain, Nicolas Roussel, Géry Casiez, and Anatole Lécuyer. 2018. Towards BCI-based interfaces for augmented reality: Feasibility, design and evaluation. IEEE Trans. Vis. Comput. Graph. (2018).

[198] Hakim Si-Mohammed, Ferran Argelaguet Sanz, Géry Casiez, Nicolas Roussel, and Anatole Lécuyer. 2017. Braincomputer interfaces and augmented reality: A state of the art. In Proceedings of the Graz Brain-Computer Interface Conference. 
[199] Hannah Slay, Bruce Thomas, and Rudi Vernik. 2002. Tangible user interaction using augmented reality. In Proceedings of the 3rd Australasian Conference on User Interfaces, Volume 7 (AUIC'02). Australian Computer Society, Inc., 13-20.

[200] R. S. Soundariya and R. Renuga. 2017. Eye movement based emotion recognition using electrooculography. In Proceedings of the 2017 Innovations in Power and Advanced Computing Technologies (i-PACT'17). IEEE, 1-5.

[201] Cometa srl. 2019. PicoEMG. Retrieved October 17, 2019 from https://www.cometasystems.com/products/picoemg.

[202] Liz Stinson. 2019. Stop the Endless Scroll. Delete Social Media from Your Phone. Retrieved October 21, 2019 from https://www.wired.com/story/rants-and-raves-desktop-social-media/.

[203] Dag Svanæs. 2013. Interaction design for and with the lived body: Some implications of merleau-ponty's phenomenology. ACM Trans. Comput.-Hum. Interact. 20, 1, Article 8 (Apr. 2013), 30 pages. https://doi.org/10.1145/2442106.2442114

[204] Daisuke Tamaki, Hiromi Fujimori, and Hisaya Tanaka. 2019. An interface using electrooculography with closed eyes. In Proceedings of the International Symposium on Affective Science and Engineering (ISASE'19). Japan Society of Kansei Engineering, 1-4.

[205] Zied Tayeb, Nicolai Waniek, Juri Fedjaev, Nejla Ghaboosi, Leonard Rychly, Christian Widderich, Christoph Richter, Jonas Braun, Matteo Saveriano, Gordon Cheng, et al. 2018. Gumpy: A python toolbox suitable for hybrid braincomputer interfaces. F. Neur. Eng. 15, 6 (2018), 065003.

[206] TMSi. 2019. Small Wearable Amplifier System. Retrieved October 17, 2019 from https://www.tmsi.com/products/ mobi/.

[207] Boris B. Velichkovsky, Mikhail A. Rumyantsev, and Mikhail A. Morozov. 2014. New solution to the midas touch problem: Identification of visual commands via extraction of focal fixations. Proc. Comput. Sci. 39 (2014), 75-82.

[208] Mélodie Vidal, Andreas Bulling, and Hans Gellersen. 2011. Analysing EOG signal features for the discrimination of eye movements with wearable devices. In Proceedings of the 1st International Workshop on Pervasive Eye Tracking \& Mobile Eye-based Interaction. 15-20.

[209] Oleg Špakov and Päivi Majaranta. 2012. Enhanced gaze interaction using simple head gestures. In Proceedings of the 2012 ACM Conference on Ubiquitous Computing (UbiComp'12). Association for Computing Machinery, New York, NY, 705-710. https://doi.org/10.1145/2370216.2370369

[210] Michael Wand and Jürgen Schmidhuber. 2016. Deep neural network frontend for continuous EMG-based speech recognition. In Proceedings of the Conference of the International Speech Communication Association (INTERSPEECH'16). 3032-3036.

[211] Meng Wang, Renjie Li, Ruofan Zhang, Guangye Li, and Dingguo Zhang. 2018. A wearable SSVEP-Based BCI system for quadcopter control using head-mounted device. IEEE Access 6 (2018), 26789-26798.

[212] Yuntao Wang, Jianyu Zhou, Hanchuan Li, Tengxiang Zhang, Minxuan Gao, Zhuolin Cheng, Chun Yu, Shwetak Patel, and Yuanchun Shi. 2019. FlexTouch: Enabling large-scale interaction sensing beyond touchscreens using flexible and conductive materials. Proc. ACM Interact. Mob. Wear. Ubiq. Technol. 3, 3, Article 109 (Sep. 2019), 20 pages. https: //doi.org/10.1145/3351267

[213] Daniel Wigdor and Dennis Wixon. 2011. Brave NUI World: Designing Natural User Interfaces for Touch and Gesture (1st ed.). Morgan Kaufmann Publishers Inc., San Francisco, CA.

[214] Woontack Woo. 2018. Augmented human: Augmented reality and beyond. In Proceedings of the 3rd International Workshop on Multimedia Alternate Realities (AltMM'18). Association for Computing Machinery, New York, NY, 1. https://doi.org/10.1145/3268998.3268999

[215] Shang-Lin Wu, Lun-De Liao, Shao-Wei Lu, Wei-Ling Jiang, Shi-An Chen, and Chin-Teng Lin. 2013. Controlling a human-computer interface system with a novel classification method that uses electrooculography signals. IEEE Trans. Biomed. Eng. 60, 8 (2013), 2133-2141.

[216] Yang Xiao, Junsong Yuan, and Daniel Thalmann. 2013. Human-virtual human interaction by upper body gesture understanding. In Proceedings of the 19th ACM Symposium on Virtual Reality Software and Technology (VRST'13). Association for Computing Machinery, New York, NY, 133-142. https://doi.org/10.1145/2503713.2503727

[217] Minpeng Xu, Xiaolin Xiao, Yijun Wang, Hongzhi Qi, Tzyy-Ping Jung, and Dong Ming. 2018. A brain-computer interface based on miniature-event-related potentials induced by very small lateral visual stimuli. IEEE Trans. Biomed. Eng. 65, 5 (2018), 1166-1175.

[218] Zheer Xu, Pui Chung Wong, Jun Gong, Te-Yen Wu, Aditya Shekhar Nittala, Xiaojun Bi, Jürgen Steimle, Hongbo Fu, Kening Zhu, and Xing-Dong Yang. 2019. TipText: Eyes-free text entry on a fingertip keyboard. In Proceedings of the 32nd Annual ACM Symposium on User Interface Software and Technology (UIST'19). Association for Computing Machinery, New York, NY, 883-899. https://doi.org/10.1145/3332165.3347865

[219] Yukang Yan, Yingtian Shi, Chun Yu, and Yuanchun Shi. 2020. HeadCross: Exploring head-based crossing selection on head-mounted displays. Proc. ACM Interact. Mob. Wear. Ubiq. Technol. 4, 1, Article 35 (Mar. 2020), 22 pages. https: //doi.org/10.1145/3380983 
[220] Chun Yu, Ke Sun, Mingyuan Zhong, Xincheng Li, Peijun Zhao, and Yuanchun Shi. 2016. One-dimensional handwriting: Inputting letters and words on smart glasses. In Proceedings of the 2016 CHI Conference on Human Factors in Computing Systems (CHI'16). Association for Computing Machinery, New York, NY, 71-82. https://doi.org/10.1145/ 2858036.2858542

[221] Nan Yu, Wei Wang, Alex X. Liu, and Lingtao Kong. 2018. QGesture: Quantifying gesture distance and direction with wifi signals. Proc. ACM Interact. Mob. Wear. Ubiq. Technol. 2, 1 (2018), 51.

[222] Hong Zeng, Yanxin Wang, Changcheng Wu, Aiguo Song, Jia Liu, Peng Ji, Baoguo Xu, Lifeng Zhu, Huijun Li, and Pengcheng Wen. 2017. Closed-loop hybrid gaze brain-machine interface based robotic arm control with augmented reality feedback. Front. Neurorobot. 11 (2017), 60.

[223] André Zenner and Antonio Krüger. 2019. Drag:On: A virtual reality controller providing haptic feedback based on drag and weight shift. In Proceedings of the 2019 CHI Conference on Human Factors in Computing Systems (CHI'19). Association for Computing Machinery, New York, NY, 1-12. https://doi.org/10.1145/3290605.3300441

[224] Xiaolong Zhai, Beth Jelfs, Rosa H. M. Chan, and Chung Tin. 2017. Self-recalibrating surface EMG pattern recognition for neuroprosthesis control based on convolutional neural network. Front. Neurosci. 11 (2017), 379. https://doi.org/ 10.3389/fnins.2017.00379

[225] Jinhua Zhang, Baozeng Wang, Cheng Zhang, Yanqing Xiao, and Michael Yu Wang. 2019. An EEG/EMG/EOG-based multimodal human-machine interface to real-time control of a soft robot hand. Front. Neurorobot. 13 (2019).

[226] Wenxiao Zhang, Bo Han, and Pan Hui. 2017. On the networking challenges of mobile augmented reality. In Proceedings of the Workshop on Virtual Reality and Augmented Reality Network. ACM, 24-29.

[227] Wenxiao Zhang, Bo Han, and Pan Hui. 2018. Jaguar: Low latency mobile augmented reality with flexible tracking. In Proceedings of the 2018 ACM Multimedia Conference on Multimedia Conference. ACM, 355-363.

[228] Xiang Zhang, Lina Yao, Quan Z. Sheng, Salil S. Kanhere, Tao Gu, and Dalin Zhang. 2018. Converting your thoughts to texts: Enabling brain typing via deep feature learning of eeg signals. In Proceedings of the 2018 IEEE International Conference on Pervasive Computing and Communications (PerCom'18). IEEE, 1-10.

[229] Xiang Zhang, Lina Yao, Shuai Zhang, Salil Kanhere, Michael Sheng, and Yunhao Liu. 2018. Internet of things meets brain-computer interface: A unified deep learning framework for enabling human-thing cognitive interactivity. IEEE IoT f. 6, 2 (2018), 2084-2092.

[230] Yu Zhang, Tao Gu, Chu Luo, Vassilis Kostakos, and Aruna Seneviratne. 2018. FinDroidHR: Smartwatch gesture input with optical heartrate monitor. Proc. ACM Interact. Mob. Wear. Ubiq. Technol. 2, 1 (2018), 56.

[231] Xuemin Zhu, Wei-Long Zheng, Bao-Liang Lu, Xiaoping Chen, Shanguang Chen, and Chunhui Wang. 2014. EOGbased drowsiness detection using convolutional neural networks. In Proceedings of the 2014 International foint Conference on Neural Networks (IFCNN'14). IEEE, 128-134.

Received November 2019; revised February 2021; accepted March 2021 